\title{
Neural Bursting and Synchronization Emulated by Neural Networks and Circuits
}

\author{
Hairong Lin, Chunhua Wang, Chengjie Chen, Yichuang Sun, Senior Member, IEEE, Chao Zhou, Cong Xu \\ and Qinghui Hong
}

\begin{abstract}
Nowadays, research, modeling, simulation and realization of brain-like systems to reproduce brain behaviors have become urgent requirements. In this paper, neural bursting and synchronization are imitated by modeling two neural network models based on the Hopfield neural network (HNN). The first neural network model consists of four neurons, which correspond to realizing neural bursting firings. Theoretical analysis and numerical simulation show that the simple neural network can generate abundant bursting dynamics including multiple periodic bursting firings with different spikes per burst, multiple coexisting bursting firings, as well as multiple chaotic bursting firings with different amplitudes. The second neural network model simulates neural synchronization using a coupling neural network composed of two above small neural networks. The synchronization dynamics of the coupling neural network is theoretically proved based on the Lyapunov stability theory. Extensive simulation results show that the coupling neural network can produce different types of synchronous behaviors dependent on synaptic coupling strength, such as anti-phase bursting synchronization, anti-phase spiking synchronization, and complete bursting synchronization. Finally, two neural network circuits are designed and implemented to show the effectiveness and potential of the constructed neural networks.
\end{abstract}

Index Terms-Bursting firing, synchronization, neural network, bifurcation, circuit implementation.

\section{INTRODUCTION}

$\mathbf{T}$ HE HUMAN brain is a complex biological nerve network composed of a great number of interactive neurons. Based on the brain nervous topology, a simplified brain neural network, namely Hopfield neural network (HNN), was constructed by Hopfield in 1982 [1]. Owing to its brain-like dynamics, the investigation of HNN is vital in understanding the electrical activities of the brain [2]. It provides insights into the qualitative behaviors of state trajectories for the variation of the network parameters, which is conducive to better understand the dynamical behaviors in the human brain. Furthermore, such a neural network with abundant dynamics is also potentially useful for developing future brain-like computing systems [3]. Recent studies have shown that the HNN can be extensively applied in various artificial intelligence scenarios,

Manuscript received October 19, 2020. This work is supported by The Major Research Project of the National Natural Science Foundation of China (91964108), The National Natural Science Foundation of China (61971185), The Open Fund Project of Key Laboratory in Hunan Universities (18K010). (Corresponding author: Chunhua Wang.)

Hairong Lin, Chunhua Wang, Chao Zhou, Cong Xu, and Qinghui Hong are with the College of Computer Science and Electronic Engineering, Hunan University, Changsha, 410082, China. (wch1227164@hnu.edu.cn)

Chengjie Chen is with School of Microelectronics and Control Engineering, Changzhou University, Changzhou, 213164, China.

Yichuang Sun is with the School of Engineering and Computer Science, University of Hertfordshire, Hatfield AL10 9AB, U.K. such as associative memory [4], machine learning [5], image processing [6]. Hence, the HNNs with complex dynamical behaviors have attracted increasing attention because of its potential significance and wide applications.

In the early days, the dynamics analyses of $\mathrm{HNN}$ focused mainly on a complete HNN model with infinite neurons. Since the discovery of brain-like chaos in an HNN with six neurons [7], small HNN models have attracted considerable attention due to their abundant dynamical behaviors. Over the past decades, various chaotic behaviors including chaos [8-11] and hyperchaos [12-14] have been revealed based on different HNN models. Especially, in recent years, great progress in neuroscience and nonlinear theory has inspired an increasing enthusiasm in research on the complex dynamical behaviors in HNNs. For example, in 2016, Pham et al. [15] discovered hidden chaotic attractors in a three-neuron-based HNN by introducing a memristor synapse to replace a resistor synapse. In 2017, Bao et al. [16] revealed a complicated phenomenon of coexisting chaotic attractors in a hyperbolic-type memristor synapse based HNN. Similarly, in 2019, coexisting multiple patterns including chaotic spiking, periodic spiking, and stable resting were detected in a memristive two-neuron-based HNN [17]. And in this year, infinitely many coexisting chaotic attractors were observed in a multi-stable memristor-based HNN with four neurons [18]. Moreover, to obtain more complex chaotic dynamics, the dynamical behaviors of the HNNs under various external stimuli have been investigated. For instance, Hu et al. [19] studied an HNN with three neurons by injecting electromagnetic radiation into its one neuron. Their research results show that the complex phenomenon of coexistence of different types of attractors can be observed under electromagnetic radiation. Inspired by their research, Lin et al. [20-22] deeply researched the influence of various external stimuli on chaotic dynamics in the HNNs. Some more intricate chaotic behaviors like attractor transition [20], extreme multistability [21], and multi-scroll attractors [22] are discovered from the HNNs.

As we all know, neural bursting is an important phenomenon in the human brain. The bursting firing universally exists in endocrine cells and the central nervous system [23], which appears when oscillation alternates between the quiescent state and the repetitive spiking firings. It is also regarded as a kind of key firing activity in keeping the normal function of the nervous system [24]. In the past decades, the bursting dynamics like periodic bursting, stochastic bursting, and chaotic bursting has been widely investigated in various single neuron models, such as Hindmarsh-Rose (HR) model [25-26], Morris- 
Lecar (ML) model [27-28], FitzHugh-Nagumo (FHN) model [29], and Chay model [30]. Nevertheless, due to the influence between neurons, the bursting firing is rarely observed in the neural networks with multiple neurons. Recently, $\mathrm{Xu}$ et al. [31] found the periodic bursting firing in a two-neuronbased non-autonomous memristive $\mathrm{HNN}$ by introducing an external sinusoidal input current. Additionally, Njitacke and his team [32-33] also observed periodic and chaotic bursting firings in a three-neuron-based HNN and a four-neuron-based HNN, respectively. However, more complex bursting dynamics including multiple burstings, coexisting multiple burstings, stochastic bursting, coexisting stochastic burstings, and coexisting chaotic burstings have not been reported in the previous researches for the Hopfield-type neural networks.

Synchronization means that two or more systems adjust to each other to lead to a common dynamic behavior, which widely exists in biological neural systems [34]. There is evidence that brain activities including sleep, thinking, and memory, are associated with synchronization at the neural level [35]. On the contrary, abnormal neural synchronization plays a crucial role in certain brain disorders like schizophrenia, epilepsy, and Parkinson's [36]. Besides, the neural network models with a synchronization phenomenon are of great importance for their potential applications. Therefore, the study on the synchronization of neural networks can not only promote the understanding of brain dynamics but also help to provide technical support for practical applications of neural network models. Indeed, during the past few decades, many investigations have been devoted to achieving the synchronization of neural networks by using various control approaches [37-41]. There are also some works investigating the phenomenon of firing synchronization in coupled bineuron networks in recent years [42-45]. For example, in [46], bursting synchronization of two coupled ML neurons under electromagnetic radiation was studied. Chaotic firing synchronization and synchronization transition of memristorbased coupled HR neurons was investigated in [47]. However, to the best of our knowledge, up to now, there are few results on the firing synchronization of two coupled neural networks with multiple neurons.

Motivated by the above discussions, this paper studies the dynamics of neural bursting and synchronization based on two neural network models with multiple neurons. Firstly, we construct a small neural network model with four neurons. The bursting dynamics is revealed by using standard nonlinear theory and numerical analysis methods. Then, we further model a coupling neural network based on two small neural networks. The synchronous behavior of the coupling neural network is theoretically proved using the Lyapunov stability theory and numerically simulated by the MATLAB tool. Finally, the phenomena of bursting and synchronization are demonstrated on the designed neural network circuits.

The rest of this paper is organized as follows. In Section II, the small neural network model is proposed, and some basic dynamical features are analyzed. In Section III, the bursting dynamics of the small neural network is revealed. In Section IV, a coupling neural network is presented based on two small neural networks, and its synchronization dynamics is discussed. In Section V, two neural network circuits are implemented physically. Section VI summarizes the full text.

\section{SMALL NEURAL NETWORK WITH FOUR NEURONS}

\section{A. Model construction}

Hopfield neural network is usually used to describe and imitate the dynamical behavior of the brain nervous system. An original Hopfield neural network which is made up of $n$ neurons can be described by [1]

$$
C_{i} \dot{x}_{i}=-x_{i} / R_{i}+\sum_{j=1}^{n} w_{i j} \tanh \left(x_{j}\right)+I_{i} \quad\left(i, j \in N^{*}\right),
$$

where $C_{i}, R_{i}$, and $x_{i}$ are considered as the membrane capacitance, membrane resistance, and membrane voltage of the neuron $i$, respectively. $\tanh ($.$) stands for the neuron activation$ function, and $w_{i j}$ denotes the synaptic weight coefficient characterizing the connection strength between neuron $i$ and neuron $j$. Additionally, $I_{i}$ represents an external input current. It should be pointed out that the dynamical behavior of the HNN heavily depends on its synaptic weight values, and the research of dynamics on a neural network with different synaptic weight coefficients is significant and valuable. For example, the HNN with four neurons in [12] generates hyperchaotic behavior, whereas the same four-neuron-based HNN with the different synaptic weights in [33] exhibits coexisting chaotic behaviors. These different dynamical behaviors are crucial for emulating the different neural dynamics of biological nervous systems. Now, we use the trial and error method to derive some appropriate synaptic weight coefficients, and a small neural network with four neurons can be constructed, as shown in Fig.1, where $N_{1}, N_{2}, N_{3}$, and $N_{4}$ are four neurons.

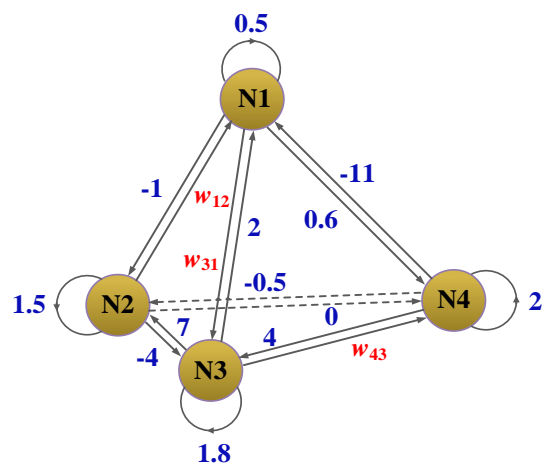

Fig. 1: Connection topology of the small neural network with four neurons

Assuming $C_{i}=1, R_{i}=1, I_{i}=0$, the presented neural network can be mathematically modeled as follows:

$$
\left\{\begin{aligned}
\dot{x_{1}} & =-x_{1}+0.5 \tanh \left(x_{1}\right)+w_{12} \tanh \left(x_{2}\right)+2 \tanh \left(x_{3}\right) \\
& \quad-11 \tanh \left(x_{4}\right) \\
\dot{x_{2}} & =-x_{2}-\tanh \left(x_{1}\right)+1.5 \tanh \left(x_{2}\right)+7 \tanh \left(x_{3}\right) \\
& \quad 0.5 \tanh \left(x_{4}\right) \\
\dot{x_{3}} & =-x_{3}+w_{31} \tanh \left(x_{1}\right)-4 \tanh \left(x_{2}\right)+1.8 \tanh \left(x_{3}\right) \\
& +4 \tanh \left(x_{4}\right) \\
\dot{x_{4}} & =-x_{4}+0.6 \tanh \left(x_{1}\right)+w_{43} \tanh \left(x_{3}\right)+2 \tanh \left(x_{4}\right)
\end{aligned}\right.
$$

where the synaptic weight coefficients $w_{12}, w_{31}$, and $w_{43}$ are regarded as three adjustable system parameters. 
TABLE I: Adjustable parameters, eigenvalues of zero equilibrium points, corresponding stabilities, and bursting dynamics

\begin{tabular}{|c|c|c|c|c|c|}
\hline$w_{12}$ & $w_{31}$ & $w_{43}$ & Eigenvalues & Stabilities & Bursting patterns \\
\hline 7 & 3 & 0.21 & $(2.3468,2.1482,-1.3475 \pm 6.3692 \mathrm{j})$ & Unstable saddle-focus & Periodic bursting \\
\hline 7 & 3 & 0.22 & $(2.2470 \pm 0.0355 \mathrm{j},-1.3470 \pm 6.3649 \mathrm{j})$ & Unstable focus & Periodic bursting \\
\hline 4 & 0.81 & -0.4 & $(0.9000 \pm 1.6720 \mathrm{j}, 0.0001 \pm 5.9602 \mathrm{j})$ & Unstable focus & Stochastic bursting \\
\hline 4 & 0.82 & -0.4 & $(0.9049 \pm 1.6721 \mathrm{j},-0.0049 \pm 5.9593 \mathrm{j})$ & Unstable focus & Stochastic bursting \\
\hline-130 & -0.1 & 0.15 & $(7.4515 \pm 0.3745 \mathrm{j},-6.5515 \pm 1.1985 \mathrm{j})$ & Unstable focus & Chaotic bursting \\
\hline-131 & -0.1 & 0.15 & $(7.6252,7.3261,-6.5756 \pm 1.1298 \mathrm{j})$ & Unstable saddle-focus & Chaotic bursting \\
\hline-139 & -0.1 & 0.15 & $(8.8203,6.5100,-6.8846,-6.6457)$ & Unstable saddle-node & Chaotic bursting \\
\hline
\end{tabular}

\section{B. Equilibria and stability analysis}

The equilibria of the small neural network and their stabilities are discussed by theoretical and numerical analysis methods. Define $E=(x, y, z, u)$ is an equilibrium point of small neural network (2). Letting the left side of Equation (2) to 0 , the equilibrium point can be solved from the following equation as

$$
\left\{\begin{array}{l}
-x+0.5 \tanh (x)+w_{12} \tanh (y)+2 \tanh (z)-11 \tanh (u) \\
=0 \\
-y-\tanh (x)+1.5 \tanh (y)+7 \tanh (z)-0.5 \tanh (u) \\
=0 \\
-z+w_{31} \tanh (x)-4 \tanh (y)+1.8 \tanh (z)+4 \tanh (u) \\
=0 \\
-u+0.6 \tanh (x)+w_{43} \tanh (z)+2 \tanh (u) \\
=0
\end{array}\right.
$$

MATLAB numerical calculations show that there is only a zero solution in Equation (3). That is to say, the neural network (2) has a zero equilibrium point $E_{0}=(0,0,0,0)$. The Jacobian matrix at the zero equilibrium point can be derived by

$$
J=\left[\begin{array}{llll}
\frac{\partial \dot{x}}{\partial x} & \frac{\partial \dot{x}}{\partial y} & \frac{\partial \dot{x}}{\partial z} & \frac{\partial \dot{x}}{\partial u} \\
\frac{\partial \dot{y}}{\partial x} & \frac{\partial \dot{y}}{\partial y} & \frac{\partial \dot{y}}{\partial z} & \frac{\partial \dot{y}}{\partial u} \\
\frac{\partial \dot{z}}{\partial x} & \frac{\partial \dot{z}}{\partial y} & \frac{\partial \dot{z}}{\partial z} & \frac{\partial \dot{z}}{\partial u} \\
\frac{\partial \dot{u}}{\partial x} & \frac{\partial \dot{u}}{\partial y} & \frac{\partial \dot{u}}{\partial z} & \frac{\partial \dot{u}}{\partial u}
\end{array}\right]
$$

$$
=\left[\begin{array}{llll}
-1+0.5 \mathrm{~s}_{x} & w_{12} \mathrm{~s}_{y} & 2 \mathrm{~s}_{z} & -11 \mathrm{~s}_{u} \\
-\mathrm{s}_{x} & -1+1.5 \mathrm{~s}_{y} & 7 \mathrm{~s}_{z} & -0.5 \mathrm{~s}_{u} \\
w_{31} \mathrm{~s}_{x} & -4 \mathrm{~s}_{y} & -1+1.8 \mathrm{~s}_{z} & 4 \mathrm{~s}_{u} \\
0.6 \mathrm{~s}_{x} & 0 & w_{43} \mathrm{~s}_{z} & -1+2 \mathrm{~s}_{u}
\end{array}\right]
$$

where $\quad s_{x}=\operatorname{sech}^{2}(x), \quad s_{y}=\operatorname{sech}^{2}(y), \quad s_{z}=\operatorname{sech}^{2}(z), \quad$ and $s_{u}=\operatorname{sech}^{2}(u)$. For the zero equilibrium point $E_{0}=(0,0$, $0,0)$, the characteristic polynomial equation is educed as

$$
\begin{aligned}
P(\lambda)= & \operatorname{det}\left(\lambda I_{E}-J\right) \\
= & \lambda^{4}-1.8 \lambda^{3}+\left(w_{12}-2 w_{31}+4 w_{43}+21.95\right) \lambda^{2} \\
& +\left(3 w_{31}-2.1 w_{12}+2 w_{43}-7 w_{12} w_{31}-11 w_{31} w_{43}\right. \\
& -8.17) \lambda+17.84 w_{12}-w_{31}-44 w_{43}+7 w_{12} w_{31} \\
& +4 w_{12} w_{43}+5.5 w_{31} w_{43}-0.5 w_{12} w_{31} w_{43}-193.64 \\
= & 0
\end{aligned}
$$

where $I_{E}$ is a four-order unit matrix. According to RouthHurwitz criteria, the zero equilibrium point is always unstable. With help of MATLAB numerical calculation for different parameters $w_{12}, w_{31}$, and $w_{43}$, the type of zero equilibrium points, and the generating firing patterns in neural network (2) are further studied. Extensive numerical simulations show that when $w_{12}=7, w_{31}=3$, the zero equilibrium point is an unstable saddle-focus for $-0.7 \leq w_{43} \leq 0.21$, and while the zero equilibrium point is translated to unstable focus for $0.22 \leq w_{43} \leq 1.5$. Under this condition, the neural network generates periodic bursting behavior. When $w_{12}=4, w_{43}=-0.4$, for $-0.5 \leq w_{31} \leq 5$ the zero equilibrium point is always an unstable focus leading to the emergence of stochastic bursting. And when $w_{31}=-$ $0.1, w_{43}=0.15$, the type of the zero equilibrium point include three cases, namely unstable focus $\left(-10 \leq w_{12} \leq-130\right)$, unstable saddle-focus $\left(-131 \leq w_{12} \leq-139\right)$, and unstable saddle-node ($\left.139 \leq w_{12} \leq-1000\right)$. Although there are three different types of zero equilibrium points the small neural network always generates chaotic bursting in this case.

Table I gives some typical values of parameters, eigenvalues of zero equilibrium points, corresponding stabilities, and the generating bursting dynamics of the small neural network. The results in Table I demonstrate that the neural network has multiple different types of unstable zero equilibrium points. Furthermore, the neural network generates three types of bursting dynamics including periodic bursting, stochastic bursting, and chaotic bursting under different values of parameters $w_{12}$, $w_{31}$, and $w_{43}$. Thus the bursting dynamics can be further studied by selecting these three parameters as adjustable parameters.

\section{BURSTING DYNAMICS IN THE SMALL NEURAL NETWORK}

In this section, the bursting dynamics of the proposed neural network with four neurons are revealed by using basic dynamic analysis methods including bifurcation diagrams, Lyapunov exponents, time series, and phase plots. And all the numerical simulations are done in MATLAB R2017a with the ODE45 algorithm. Additionally, the start time, the time step, and the time length are set as 500, 0.01, and 3000, respectively.

Before researching the bursting dynamics of the neural network, it is necessary to introduce the basic definition of various bursting patterns [24-25]. In general, in neurons or neural networks, if the trajectory of the membrane potential alternates between a quiescent state and repetitive spiking, such behavior is considered as a bursting pattern. Meanwhile, the bursting pattern can be divided into periodic bursting, stochastic bursting, and chaotic bursting. Among them, periodic bursting including $m\left(m \in N^{*}\right)$ spikes per burst is said to be period- $m$ bursting. If the trajectory of bursting firing is a stochastic transition between bursting and spiking, the bursting pattern is called stochastic bursting. For the chaotic bursting, except for the period- $m$ bursting, there exist other kinds of bursting with different numbers or amplitudes of spikes. Additionally, from the point of view of chaos [48-49], both stochastic bursting and chaotic bursting are also chaotic behaviors, but they are different from chaos firing that is a completely chaotic oscillation [50]. 


\section{A. Multiple periodic bursting firings}

Setting the adjustable parameters $w_{12}=7$ and $w_{31}=3$, the $w_{43}$-based bifurcation diagram is plotted in Fig.2 under initial states $\left(x_{1}(0), x_{2}(0), x_{3}(0), x_{4}(0)\right)=(0.1,0,0,0.1)$, where $x_{1}$ peak is the peaks of the membrane potential $x_{1}$. It can be seen from Fig. 2 that the neural network can generate abundant periodic behaviors with different periods in a wide region $w_{43} \in(-0.71,1.2)$. And when $w_{43} \geq 1.2$, the neuarl network exhibits periodic spiking firing. More importantly, simulation results show that these periodic behaviors are periodic bursting firings with different spikes per burst. Also, the number of spikes per burst is gradually increased with the decrease of $w_{43}$. That is, multiple periodic bursting patterns with different spikes per burst can be observed under different intensity of synaptic weight $w_{43}$. As shown in Fig.3, by selecting different $w_{43}$, period- $m(m=4,5,6,7,9,10)$ bursting patterns can be generated from the presented neural network. And we have confirmed that more bursting patterns with different spikes per burst can be found via selecting more $w_{43}$. Besides, the time series of four membrane potentials $x_{1}-x_{4}$ are given in Fig.4. It can be seen that the four neurons simultaneously produce bursting firings, which means that the presented small neural network really generates bursting behavior.

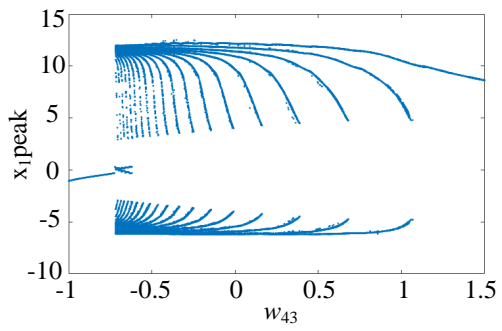

Fig. 2: The $w_{43}$-dependent bifurcation diagram with $w_{12}=7$, $w_{31}=3$ and initial states $(0.1,0,0,0.1)$.

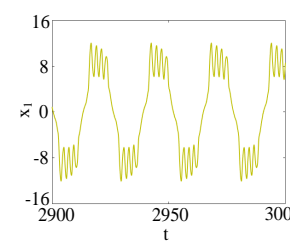

(a)

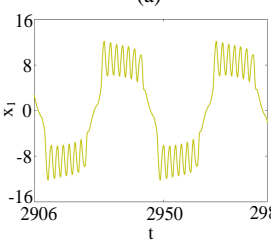

(d)

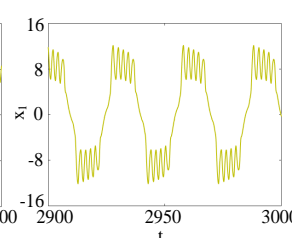

$\mathrm{t}$
(b)

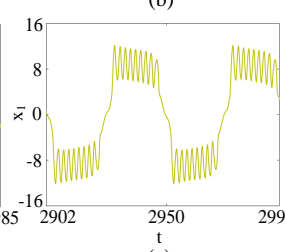

(e)

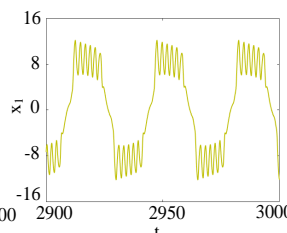

(c)

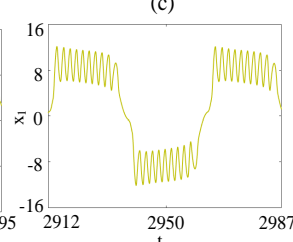

$\mathrm{t}$
(f)
Fig. 3: Multiple periodic burstings with different spikes per burst in the neural network with $w_{12}=7, w_{31}=3$, and initial states $(0.1,0,0,0.1)$. (a) period-4 bursting with $w_{43}=0.18$. (b) period-5 bursting with $w_{43}=0$. (c) period-6 bursting with $w_{43}=-0.15$. (d) period- 7 bursting with $w_{43}=-0.25$. (e) period -9 bursting with $w_{43}=-0.4$. (f) period-10 bursting with $w_{43}=-0.45$.

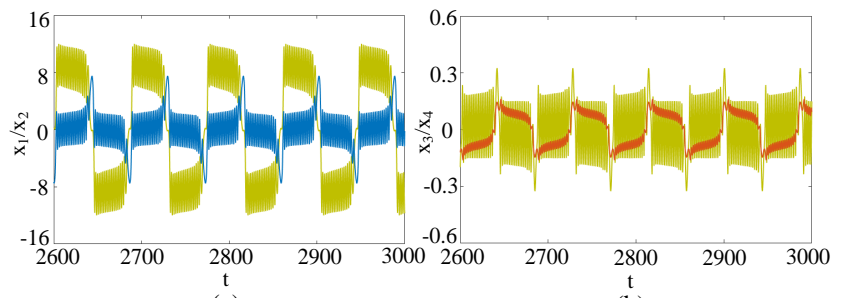

(a)

(b)

Fig. 4: Synchronous bursting firings of neurons in the small neural network. (a) membrane potential $x_{1}$ colored in yellow, and membrane potential $x_{2}$ colored in blue. (b) membrane potential $x_{3}$ colored in yellow, and membrane potential $x_{4}$ colored in orange.

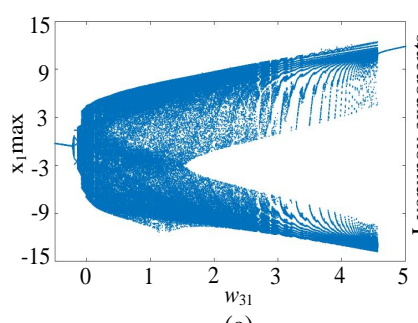

(a)

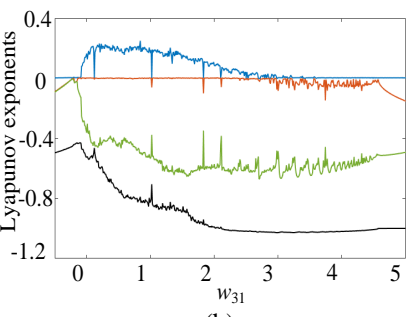

(b)
Fig. 5: The $w_{31}$-dependent dynamics with $w_{12}=4, w_{43}=-0.4$ and initial states $(0.1,0,0,0.1)$. (a) bifurcation diagram. (b) First four Lyapunov exponents.

\section{B. multiple sets of coexisting bursting firings}

Coexisting behaviors in neural systems have become a significant research topic and received wide attention. Particularly, the phenomenon of coexisting bursting firings means that there are two different bursting behaviors in the same neural system under two different initial conditions. In this subsection, the phenomenon of multiple sets of coexisting bursting firings is discovered by investigating the adjustable parameter $w_{31}$. When the synaptic weight coefficients $w_{12}=4, w_{43}=-0.4$, initial states $\left(x_{1}(0), x_{2}(0), x_{3}(0), x_{4}(0)\right)=(0.1,0,0,0.1)$, and $w_{31}$ is increased from -0.5 to 5 , the bifurcation diagram of the $w_{31}$ on the $x_{1}$-plane are depicted in Fig.5(a). And the corresponding first four Lyapunov exponents are shown in Fig.5(b). In Fig.5(a), when the $w_{31}$ increases from -0.5 , the dynamical orbit of the neural network begins with periodic spiking firing and evolves to the quasi-period oscillation at $w_{31}=-0.2$. Thereafter, the orbits break into chaos firing pattern at $w_{31}=0$ until $w_{31}=0.3$. Interestingly, as $w_{31}$ increases further, the chaos firing is gradually transformed into stochastic bursting firing until $w_{31}=1.2$. Then the stochastic bursting firing enters into chaotic bursting firing and finally ends at $w_{31}=2.6$. What follows is periodic bursting firing in the interval $w_{31} \in(2.7$, 4.5). The corresponding Lyapunov exponents in Fig.5(b) are consistent with the dynamical behaviors on the bifurcation diagram in Fig.5(a). For different strengths of the synaptic weight $w_{31}$, the phenomena of periodic spiking, chaos firing, stochastic bursting, chaotic bursting, and periodic bursting can be obtained in the presented small neural network, as shown in Fig.6. What is more important, under these parameters, for 
different initial states $(0,1,0,0,0.1)$ and $(-0.1,0,0,-0.1)$, the neural network exhibits coexisting stochastic bursting firings, coexisting chaotic bursting firings, and coexisting periodic bursting firings, as shown in Fig.7. Such complex bursting dynamics means the neural network can generate multiple sets of coexisting bursting firings.

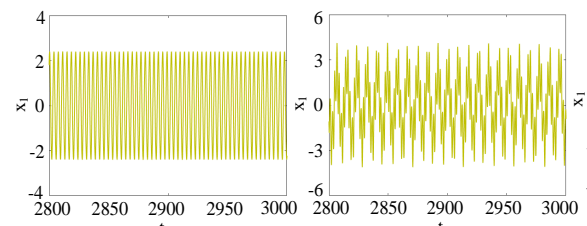

(a)

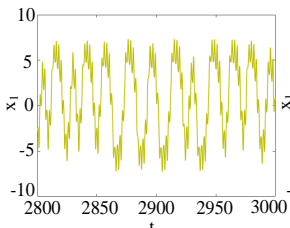

(d) (b)

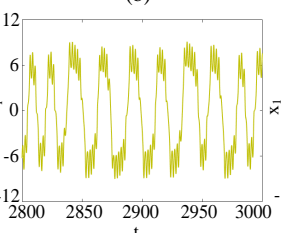

(e)

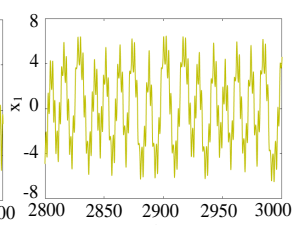

(c)

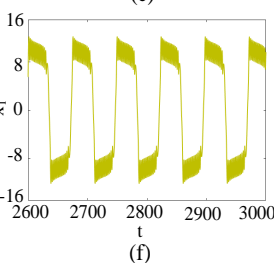

Fig. 6: Firing patterns of the neural network for different values of $w_{31}$. (a) periodic spiking firing with $w_{31}=-0.5$. (b) quasi-periodic spiking firing with $w_{31}=-0.1$. (c) chaos firing with $w_{31}=0.1$. (d) stochastic bursting firing with $w_{31}=0.4$. (e) chaotic bursting firing with $w_{31}=1.5$. (f) periodic bursting firing with $w_{31}=4$.

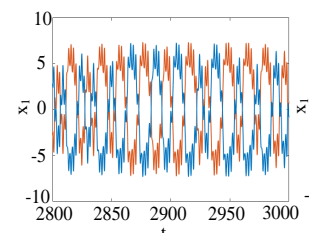

(a)

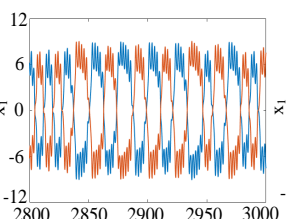

(b)

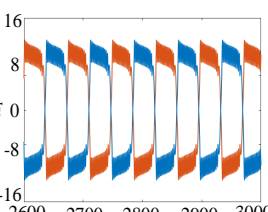

(c)
Fig. 7: Multiple sets of coexisting bursting firings in the HNN with initial states $(0.1,0,0,0.1)$ colored in red and $(-0.1$, $0,0,-0.1)$ colored in blue. (a) coexisting stochastic bursting firings with $w_{31}=0.4$. (b) coexisting chaotic bursting firings with $w_{31}=1.5$. (c) coexisting periodic bursting firings with $w_{31}=4$.

\section{Multiple bursting firings with different amplitudes}

The amplitude control plays an important role in dynamical systems and has valuable applications [51-52]. But such a phenomenon has not been observed in neural networks. It is wonderful that the presented neural network can generate periodic and chaotic bursting firings with different amplitudes under different strengths of parameter $w_{12}$. That is to say, the amplitude of the generated neural signals is freely controlled without using amplifiers, which undoubtedly extends its range of application. In the following analyses, the synaptic weight coefficients $w_{43}=0.15$, and initial states $(0.1,0,0,0.1)$ are fixed, and when $w_{31}=-0.23$ or -0.1 , the adjustable parameter $w_{12}$ is used to realize amplitude control. First, for $w_{31}=-0.23$, extensive numerical simulations show that infinitely many periodic bursting patterns with different amplitudes can be generated from the neural network under different $w_{12}$. For example, when the adjustable parameter $w_{12}=-10,-40,-80$, $120,-160$, and -200 , respectively, six periodic bursting firings with different amplitudes and corresponding periodic attractors with different phase-amplitudes on the $x$-axis can be observed as shown in Fig.8(a) and (b).

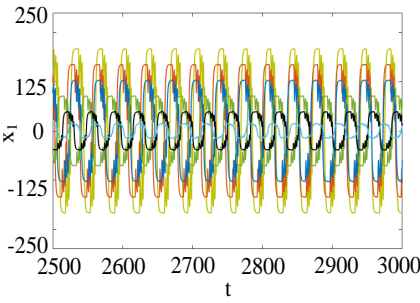

(a)

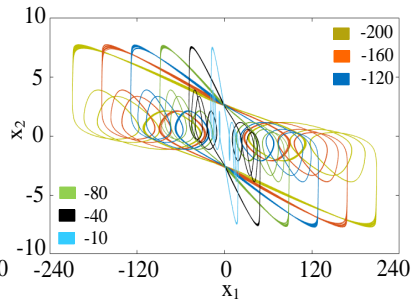

(b)
Fig. 8: Periodic bursting firings with different amplitudes in the neural network with $w_{31}=-0.23, w_{43}=0.15$, and different $w_{12}$. (a) Time series (b) phase portrait.

Moreover, for $w_{31}=-0.1$, when six different values of $w_{12}$ are selected, six chaotic bursting firings with different amplitudes can be observed from the neural network. And the time series of the six chaotic bursting firings and corresponding chaotic attractors are given in Fig.9(a) and (b). We can see from Fig.8 and Fig.9 that the amplitude of the membrane potential $x_{1}$ is more and more larger with the decrease of the $w_{12}$. To further confirm this feature, for $w_{12} \in(-1000$, 10 ), when $w_{31}=-0.23$ and -0.1 , two bifurcation diagrams about the $w_{12}$ are respectively plotted in Fig. 10(a) and Fig.10(b). Fig.10 directly illustrates two important phenomena: (i) the small neural network generates periodic bursting firing and chaotic bursting firing; (ii) the amplitude of the bursting firing can be freely adjusted by the synaptic weight coefficient $w_{12}$.

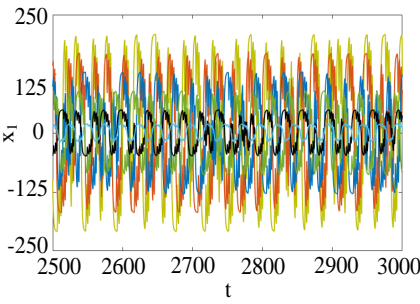

(a)

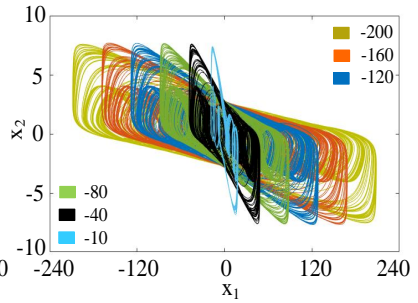

(b)
Fig. 9: Chaotic bursting firings with different amplitudes in the neural network $w_{31}=-0.1, w_{43}=0.15$, and different $w_{12}$. (a) Time series (b) phase portrait.

\section{SYNCHRONOUS DYNAMICS IN THE COUPLING NEURAL NETWORK}

The electrical activity for synchronization widely exists in many specific areas of the brain, which plays crucial roles in the information processing and transmission between two neural networks [36]. Based on this physiological mechanism, a synaptic coupling neural network model is, thereby, constructed and the complex synchronization phenomenon of bursting firing is simulated. Fig.11 gives a concept map of 


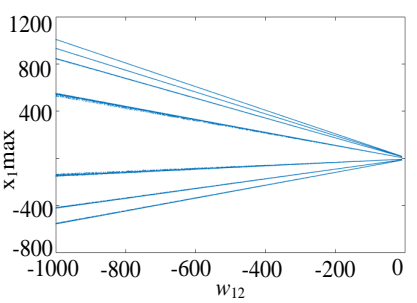

(a)

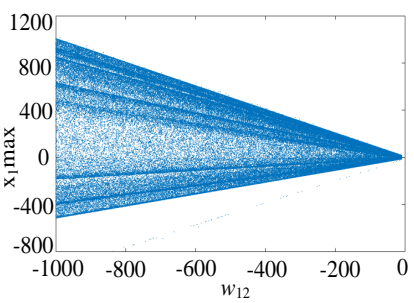

(b)
Fig. 10: The $w_{12}$-dependent bifurcation diagrams with $w_{43}=0.15$ and initial states $(0.1,0,0,0.1)$. (a) $w_{31}=-0.23$. (b) $w_{31}=-0.1$.

the coupling neural network, in which the two sub-networks represent two different nervous areas in the brain. And the two sub-networks are realized using the small neural network in Equation (2). Considering that the first sub-network has a state variable $\left(x_{1}, x_{2}, x_{3}, x_{4}\right)$ and the second sub-network has a state variable $\left(y_{1}, y_{2}, y_{3}, y_{4}\right)$, the synaptic coupling neural network model is defined by

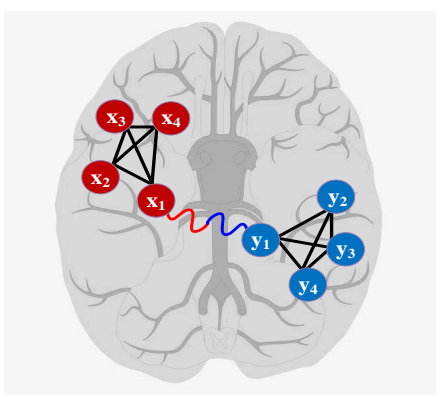

Fig. 11: Concept map of the coupling neural network.

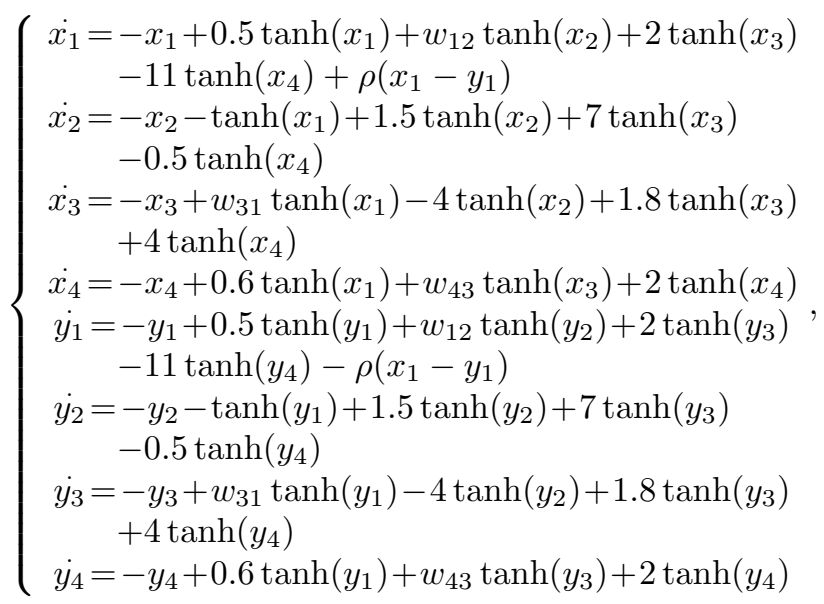

where $\rho$ denotes the synaptic coupling strength between two sub-networks.

\section{A. Stability of synchronous states and Lyapunov function}

The complete synchronization of the coupling neural network in Equation (6) occurs when the two sub-networks asymptotically exhibit identical behavior, that is, as $t \rightarrow \infty$,

$$
\left\{\begin{array}{l}
\left\|x_{1}-y_{1}\right\| \rightarrow 0 \\
\left\|x_{2}-y_{2}\right\| \rightarrow 0 \\
\left\|x_{3}-y_{3}\right\| \rightarrow 0 \\
\left\|x_{4}-y_{4}\right\| \rightarrow 0
\end{array} .\right.
$$

Generally, this synchronization solution might be stable only under some conditions. To investigate the stability of synchronized states of the coupling neural network, the Lyapunov function approach is adopted. We define the synchronization error $e\left(e_{1}, e_{2}, e_{3}, e_{4}\right)$ in the following way:

$$
e=\left\{\begin{array}{l}
e_{1}=x_{1}-y_{1} \\
e_{2}=x_{2}-y_{2} \\
e_{3}=x_{3}-y_{3} \\
e_{4}=x_{4}-y_{4}
\end{array}\right.
$$

Then, we can easily deduce the system below, which makes it possible to describe the dynamics of the synchronization error:

$$
\dot{e}=\left\{\begin{aligned}
\dot{e_{1}}= & -e_{1}+0.5\left(\tanh \left(x_{1}\right)-\tanh \left(y_{1}\right)\right) \\
& +w_{12}\left(\tanh \left(x_{2}\right)-\tanh \left(y_{2}\right)\right) \\
& +2\left(\tanh \left(x_{3}\right)-\tanh \left(y_{3}\right)\right) \\
& -11\left(\tanh \left(x_{4}\right)-\tanh \left(y_{4}\right)\right)+2 \rho e_{1} \\
\dot{e_{2}}= & -e_{2}-\left(\tanh \left(x_{1}\right)-\tanh \left(y_{1}\right)\right) \\
& +1.5\left(\tanh \left(x_{2}\right)-\tanh \left(y_{2}\right)\right) \\
& +7\left(\tanh \left(x_{3}\right)-\tanh \left(y_{3}\right)\right) \\
& -0.5\left(\tanh \left(x_{4}\right)-\tanh \left(y_{4}\right)\right) \\
\dot{e_{3}}= & -e_{3}+w_{31}\left(\tanh \left(x_{1}\right)-\tanh \left(y_{1}\right)\right) \\
& -4\left(\tanh \left(x_{2}\right)-\tanh \left(y_{2}\right)\right) \\
& +1.8\left(\tanh \left(x_{3}\right)-\tanh \left(y_{3}\right)\right) \\
& +4\left(\tanh \left(x_{4}\right)-\tanh \left(y_{4}\right)\right) \\
\dot{e_{4}}= & -e_{4}+0.6\left(\tanh \left(x_{1}\right)-\tanh \left(y_{1}\right)\right) \\
& +w_{43}\left(\tanh \left(x_{3}\right)-\tanh \left(y_{3}\right)\right) \\
& +2\left(\tanh \left(x_{4}\right)-\tanh \left(y_{4}\right)\right)
\end{aligned}\right.
$$

It is easy to obtain that the origin of $e=0$ is the equilibrium point for the error system (9). The problem of synchronization between the two sub-networks now amounts to ensuring that the error dynamics described by Equation (9) are asymptotically stable. The following theorem can be obtained

Theorem 1: The coupling neural network in (6) completely synchronizes if the error dynamical system in Equation (9) is asymptotically stable. That is, to ensure that the error dynamical system in Equation (9) is stable, in the Lyapunov sense, the time derivative of the Lyapunov function must be negative semi-definite.

Proof: We construct a continuous positive-definite Lyapunov function of the form

$$
V\left(e_{1}, e_{2}, e_{3}, e_{4}\right)=\frac{1}{2}\left(e_{1}^{2}+e_{2}^{2}+e_{3}^{2}+e_{4}^{2}\right)
$$

The time derivative of the Lyapunov function $V$ along with trajectories of the error dynamical system in Equation (9) 
yields

$$
\begin{aligned}
\frac{d V}{d t}= & \left(e_{1} \dot{e_{1}}+e_{2} \dot{e_{2}}+e_{3} \dot{e_{3}}+e_{4} \dot{e_{4}}\right) \\
= & (2 \rho-1) e_{1}^{2}+0.5 e_{1}\left(\tanh \left(x_{1}\right)-\tanh \left(y_{1}\right)\right) \\
& +w_{12} e_{1}\left(\tanh \left(x_{2}\right)-\tanh \left(y_{2}\right)\right) \\
& +2 e_{1}\left(\tanh \left(x_{3}\right)-\tanh \left(y_{3}\right)\right) \\
& -11 e_{1}\left(\tanh \left(x_{4}\right)-\tanh \left(y_{4}\right)\right) \\
& -e_{2}^{2}-e_{2}\left(\tanh \left(x_{1}\right)-\tanh \left(y_{1}\right)\right) \\
& +1.5 e_{2}\left(\tanh \left(x_{2}\right)-\tanh \left(y_{2}\right)\right) \\
& +7 e_{2}\left(\tanh \left(x_{3}\right)-\tanh \left(y_{3}\right)\right) \\
& -0.5 e_{2}\left(\tanh \left(x_{4}\right)-\tanh \left(y_{4}\right)\right) \\
& -e_{3}^{2}+w_{31} e_{3}\left(\tanh \left(x_{1}\right)-\tanh \left(y_{1}\right)\right) \\
& -4 e_{3}\left(\tanh \left(x_{2}\right)-\tanh \left(y_{2}\right)\right) \\
& +1.8 e_{3}\left(\tanh \left(x_{3}\right)-\tanh \left(y_{3}\right)\right) \\
& +4 e_{3}\left(\tanh \left(x_{4}\right)-\tanh \left(y_{4}\right)\right) \\
& -e_{4}^{2}+0.6 e_{4}\left(\tanh \left(x_{1}\right)-\tanh \left(y_{1}\right)\right) \\
& +w_{43} e_{4}\left(\tanh \left(x_{3}\right)-\tanh \left(y_{3}\right)\right) \\
& +2 e_{4}\left(\tanh \left(x_{4}\right)-\tanh \left(y_{4}\right)\right)
\end{aligned}
$$

To simplify the calculation process, let us define that

$$
\begin{aligned}
& h\left(x_{1}, x_{2}, x_{3}, x_{4}, y_{1}, y_{2}, y_{3}, y_{4}\right)= \\
& \left(0.5 e_{1}-e_{2}+w_{31} e_{3}+0.6 e_{4}\right)\left(\tanh \left(x_{1}\right)-\tanh \left(y_{1}\right)\right) \\
& +\left(w_{12} e_{1}+1.5 e_{2}-4 e_{3}\right)\left(\tanh \left(x_{2}\right)-\tanh \left(y_{2}\right)\right) \\
& +\left(2 e_{1}+7 e_{2}+1.8 e_{3}+w_{43} e_{4}\right)\left(\tanh \left(x_{3}\right)-\tanh \left(y_{3}\right)\right) \\
& -\left(11 e_{1}+0.5 e_{2}-4 e_{3}-2 e_{4}\right)\left(\tanh \left(x_{4}\right)-\tanh \left(y_{4}\right)\right)
\end{aligned}
$$

For $x_{i} \in R, \tanh \left(x_{i}\right)<1(i=1,2,3, \ldots)$, thus Equation (12) can be simplified by

$$
\begin{aligned}
h \leq & 2\left(0.5 e_{1}-e_{2}+w_{31} e_{3}+0.6 e_{4}\right) \\
& +2\left(w_{12} e_{1}+1.5 e_{2}-4 e_{3}\right) \\
& +2\left(2 e_{1}+7 e_{2}+1.8 e_{3}+w_{43} e_{4}\right) \\
& +2\left(11 e_{1}+0.5 e_{2}-4 e_{3}-2 e_{4}\right) \\
\leq & 2\left(13.5+w_{12}\right) e_{1}+16 e_{2}+2\left(6.2+w_{31}\right) e_{3} \\
& +2\left(w_{43}-1.4\right) e_{4}
\end{aligned}
$$

Then using Equation (13) put into Equation (11)

$$
\begin{aligned}
\frac{d V}{d t}= & (2 \rho-1) e_{1}{ }^{2}-e_{2}{ }^{2}-e_{3}{ }^{2}-e_{4}{ }^{2}+h \\
\leq & (2 \rho-1) e_{1}^{2}+e_{2}{ }^{2}+e_{3}{ }^{2}+e_{4}{ }^{2} \\
& +2\left(13.5+w_{12}\right) e_{1}+16 e_{2} \\
& +2\left(6.2+w_{31}\right) e_{3}+2\left(w_{43}-1.4\right) e_{4} \\
\leq & (2 \rho-1)\left(e_{1}{ }^{2}+2 s_{1} e_{1}+s_{1}{ }^{2}\right)+e_{2}{ }^{2} \\
& +2 s_{2} e_{2}+s_{2}{ }^{2}+e_{3}{ }^{2}+2 s_{3} e_{3}+s_{3}{ }^{2} \\
& +e_{4}{ }^{2}+2 s_{4} e_{4}+s_{4}{ }^{2} \\
\leq & (2 \rho-1)\left(e_{1}+s_{1}\right)^{2}+\left(e_{2}+s_{2}\right)^{2} \\
& +\left(e_{3}+s_{3}\right)^{2}+\left(e_{4}+s_{4}\right)^{2}
\end{aligned}
$$

where

$$
\left\{\begin{array}{l}
s_{1}=\left(13.5+w_{12}\right) /(1-2 \rho) \\
s_{2}=8 \\
s_{3}=6.2+w_{31} \\
s_{4}=w_{43}-1.4
\end{array} .\right.
$$

To ensure that the origin of the error dynamical system in Equation (9) is stable, in the Lyapunov sense, $\mathrm{d} V / \mathrm{dt}$ in Equation (14) should be negative semi-definite. Since two sub-neural networks have bounded trajectories, there exists a sufficiently large constant $c$. For all state variables satisfying $x_{1}-y_{1}=e_{1}<c, x_{2}-y_{2}=e_{2}<c, x_{3}-y_{3}=e_{3}<c, x_{4}-y_{4}=e_{4}<c$, thus there exists

$$
\left(c+s_{2}\right)^{2}+\left(c+s_{3}\right)^{2}+\left(c+s_{4}\right)^{2}<(1-2 \rho)\left(c+s_{1}\right)^{2} .
$$

As a result, there is

$$
\begin{aligned}
\frac{d V}{d t} \leq & (2 \rho-1)\left(e_{1}+s_{1}\right)^{2}+\left(e_{2}+s_{2}\right)^{2} \\
& +\left(e_{3}+s_{3}\right)^{2}+\left(e_{4}+s_{4}\right)^{2} \\
< & (2 \rho-1)\left(c+s_{1}\right)^{2}+\left(c+s_{2}\right)^{2} \\
& +\left(c+s_{3}\right)^{2}+\left(c+s_{4}\right)^{2} \\
< & 0
\end{aligned} .
$$

Therefore, according to Lyapunov stability theory and Barbalat's lemma, all the transverse perturbations decay to the synchronization manifold without any transient growth, i.e., one obtains

$$
\left\{\begin{array}{l}
e_{1}(t) \rightarrow 0 \\
e_{2}(t) \rightarrow 0 \\
e_{3}(t) \rightarrow 0 \\
e_{4}(t) \rightarrow 0
\end{array} .\right.
$$

as $t \rightarrow \infty$. It follows that the coupling neural network in Equation (6) synchronizes when the inequalities in Equation (16) are satisfied. This completes the proof.

\section{B. Numerical validations}

In this subsection, synchronization firings in the coupling neural network in Equation (6) is numerically confirmed by using the four-order Runge-Kutta algorithm with fixed timestep 0.01 . The adjustable parameters and initial states of two sub-networks are fixed as $w_{12}=7, w_{31}=3, w_{43}=-0.45$, $\left(x_{1}(0), x_{2}(0), x_{3}(0), x_{4}(0)\right)=(0.1,0,0,0.1)$ and $\left(y_{1}(0), y_{2}(0)\right.$, $\left.y_{3}(0), y_{4}(0)\right)=(0,0.1,0.1,0)$, respectively.Before exploring the phenomenon of synchronization in the coupling neural network, it is necessary to introduce the basic definition of various types of synchrony such as complete synchronization and anti-phase synchronization [53]. Complete synchronization is defined as a form of synchronization where the distance between the states of two coupled systems approaches zero for $t \rightarrow \infty$, i.e, the synchronization error $e \rightarrow 0$. If the state variables of two coupled systems have the same amplitude but different signs, the form of synchronization is called anti-phase synchronization.

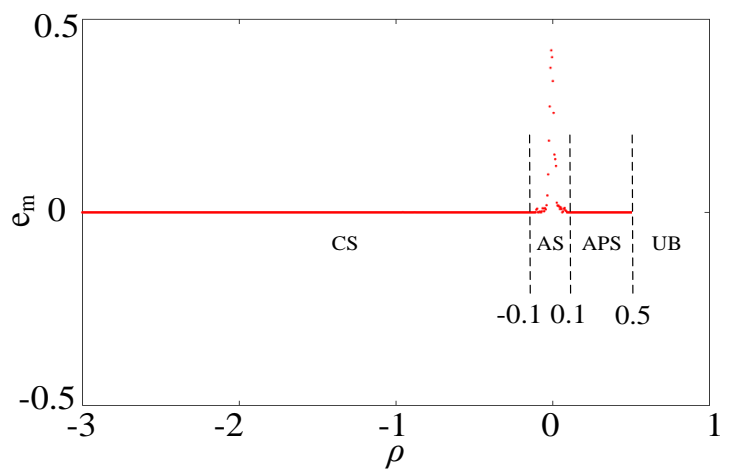

Fig. 12: Dependence of the mean synchronized error em on the coupling strength $\rho$, where the parameters and initial conditions are $w_{12}=7, w_{31}=3, w_{43}=-0.45$, and $(0.1,0,0,0.1$, $0,0.1,0.1,0)$. 


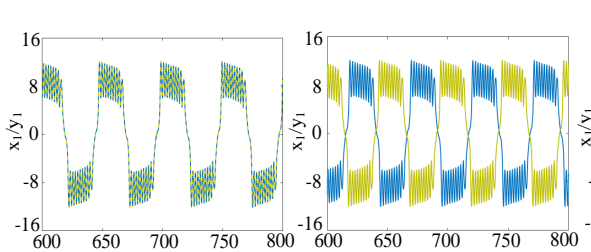

$\stackrel{\mathrm{t}}{\text { (a1) }}$

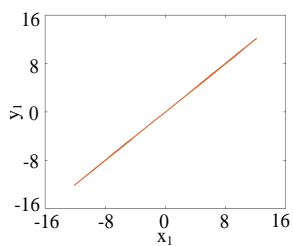

(b1) $\mathrm{t}$
(a2)

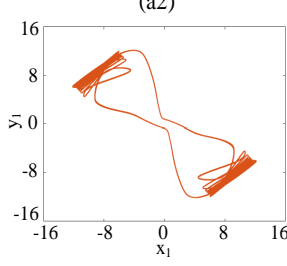

(b2)

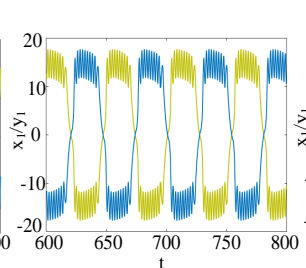

(a3)

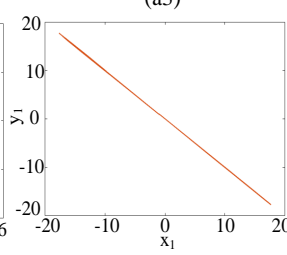

(b3)

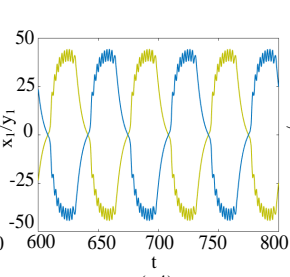

(a4)

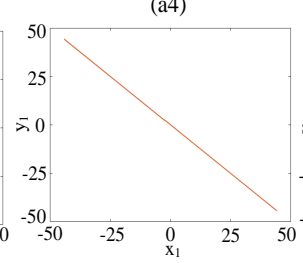

(b4)

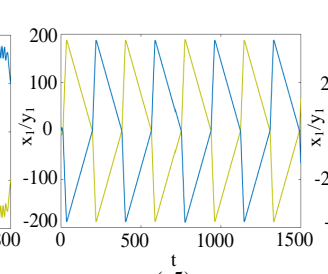

(a5)

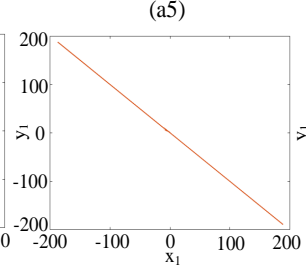

(b5)

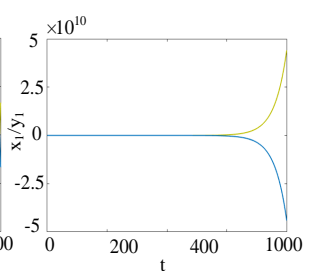

(a6)

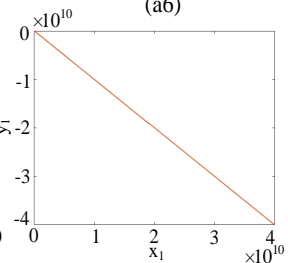

(b6)

Fig. 13: Synchronous firing activities of the coupling neural network with different coupling strengths, where $w_{12}=7, w_{31}=3$, $w_{43}=-0.45$, the tops are time series of firing behaviors, and the bottoms are synchronization transition state. (a1, b1) complete bursting synchronization with $\rho=-2$. (a2, b2) asynchronous bursting with $\rho=0$. (a3, b3) anti-phase bursting synchronization with $\rho=0.2$. (a4, b4) anti-phase bursting synchronization with $\rho=0.4$. (a5, b5) anti-phase spiking synchronization with $\rho=0.5$. (a6, b6) unbounded state with $\rho=0.51$.
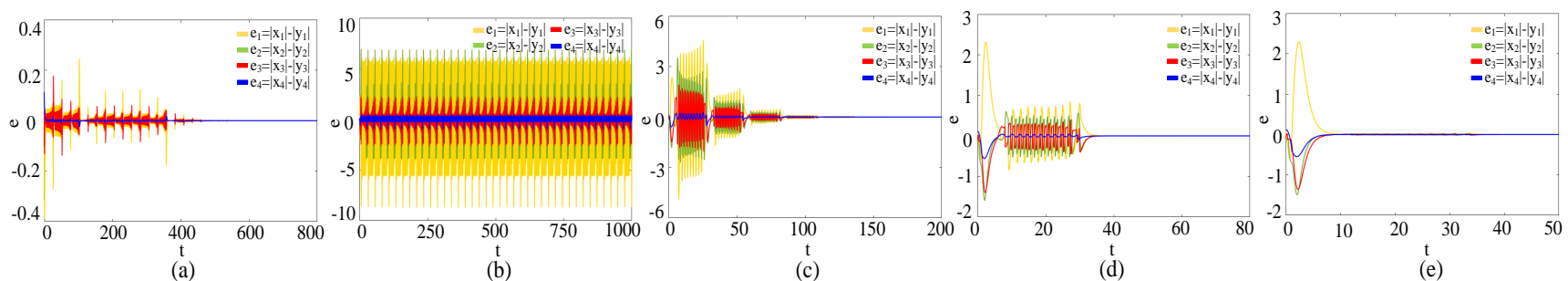

Fig. 14: Time evolution of the synchronization errors $e_{1}, e_{2}, e_{3}$, and $e_{4}$ between two coupled neural networks with different coupling strengths. (a) complete synchronization $\rho=-2$. (b) asynochronization $\rho=0$. (c) anti-phase synchronization $\rho=0.2$. (d) anti-phase synchronization $\rho=0.4$. (e) anti-phase synchronization $\rho=0.5$.

To quantitatively depict the error of two coupled neural networks, a normalized mean synchronization error $e_{m}$ can be defined as [45]:

$$
e_{m}=\frac{1}{N} \sum_{n=1}^{N} \frac{\sqrt{\sum_{i=1}^{4}\left(\left|x_{i}(n)\right|-\left|y_{i}(n)\right|\right)^{2}}}{\sqrt{\sum_{i=1}^{4}\left(x_{i}(n)^{2}+y_{i}(n)^{2}\right)}},
$$

where $x_{i}(n)$ and $y_{i}(n)$ are the $n$th sampling values with $N$ samples during a time sequence interval. Here, $e_{m}$ can be used as an index to describe synchronization behavior. With Equation (19), normalized mean synchronization error of the coupling neural network can be calculated, so that $e_{m} \rightarrow 0$ is relative to the synchronous state. For the time sequence interval $[500,1000]$ with time-step 0.01 and sampled size $N=10000$, the normalized mean synchronization error for different coupling strength $\rho$ is plotted in the $e_{m}-\rho$ plane, as shown in Fig.12. It can be seen from Fig.12 that the mean synchronization error $e_{m}$ is always equal to zero when the coupling strength $\rho \leq-0.1$. That is to say, in this case, the two coupled neural networks have a complete synchronization (CS) state. However, as $\rho$ further increases, the two coupled neural networks exhibit an asynchronous state (AS) due to $e_{m}>0$.
Interestingly, when $\rho=0.1$, the two coupled neural networks enter into an anti-phase synchronization (APS) state until $\rho=0.5$. Finally, the dynamical states of the two coupled neural networks enter into an unbounded (UB) state.

Meanwhile, the time sequences and corresponding phase diagrams in the coupling neural network with different coupling strengths are plotted in Fig.13. In Fig.13(a1), when the coupling strength is very small $(\rho=-2)$, the two coupled sub-networks present complete bursting synchronization. As $\rho$ increases to 0 , the two coupled sub-networks show an asynchronous bursting state, as shown in Fig.13(a2). As $\rho$ further increases, such as $\rho=0.2$ and $\rho=0.4$, two anti-phase bursting synchronizations with different amplitudes can be observed in the two coupled sub-networks, as shown in Fig.13(a3) and (a4), respectively. And for $\rho=0.5$, the two coupled subnetworks show anti-phase spiking synchronization, as shown in Fig.13(a5). But, when $\rho$ further increases to 0.51, the two sub-networks enter into an unbounded state, as shown in Fig.13(a6).

Furthermore, the synchronization errors of different types of synchronization are given in Fig.14. It should be noted that to better compare different types of synchronization behaviors, the synchronization error in Fig.14 is defined as the difference of the absolute value of state variables. We can see from Fig.14(a, c, d, e) that the synchronization errors of various 
types of synchronization states gradually trend to zero with the evolution of time. While the synchronization error of asynchronous state with $\rho=0$ is always greater than zero. Obviously, the results in Fig.14 are consistent with the results in Fig.13, which further shows that there exist synchronization transitions, i.e., synchronized dynamics can be switched from complete synchronization to anti-phase synchronization just by the increase of the coupling strength.

\section{CiRCUit IMPlEMENTATION AND DEMONSTRATION}

The physical implementation of neural network models is vital and necessary to develop neuromorphic hardware systems. Up to now, many neural networks have been physically realized by using analog circuits [54], digital circuits [5556], or field-programmable gate array [57]. Generally, analog neural network circuit can realize the real-time calculation and is able to reproduce the behavior of a real neural system. In this section, the proposed two neural network models are completely implemented by using basic electronic circuit elements such as diodes, resistors, capacitors, and operational amplifiers. We firstly design the circuit of the small neural network model, and its bursting behavior is demonstrated on an experimental hardware circuit. Then, the circuit of the coupling neural network model is designed and simulated in the PSPICE circuit simulation tool.

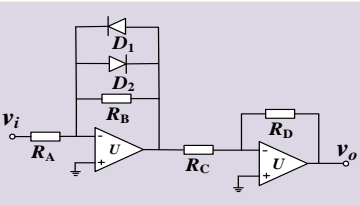

(a)

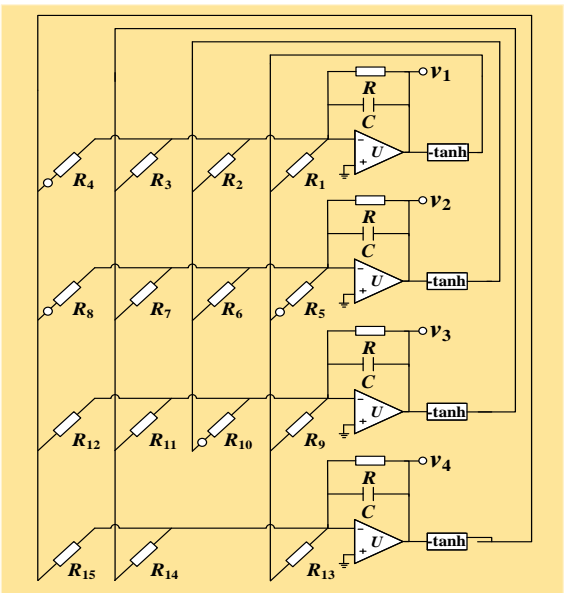

(b)

Fig. 15: Circuit structure. (a) the hyperbolic tangent function circuit. (b) the small neural network circuit.

\section{A. Design and measurement of the small neural network circuit}

Before realizing the neural network circuit, we first design an approximate hyperbolic tangent exciting function circuit [58], as shown in Fig.15(a). The hyperbolic tangent function circuit is composed of two operation amplifiers, two diodes, and four resistors, which enjoys a more simple circuit structure compared with the hyperbolic tangent circuit in traditional HNNs [16-22]. When $R_{A}=1.5 \mathrm{k} \Omega, R_{B}=0.5 \mathrm{k} \Omega, R_{C}=1 \mathrm{k} \Omega$, and $R_{D}=1.5 \mathrm{k} \Omega$, the input-output relationship of the hyperbolic tangent circuit can be described by $v_{o}=\tanh \left(v_{i}\right)$. An analog circuit implementing neural network (2) is designed for measuring neural bursting dynamics. To avoid circuit saturations, the amplitude of the membrane potential $\left(x_{1}\right.$ and $x_{2}$ ) are reduced by two times through taking the following transformations

$$
\left(x_{1}, x_{2}, x_{3}, x_{4}\right) \rightarrow\left(2 x_{1}, 2 x_{2}, x_{3}, x_{4}\right) .
$$

Equation (2) is then rewritten as

$$
\begin{aligned}
\dot{x}_{1}= & -x_{1}+0.25 \tanh \left(2 x_{1}\right)+0.5 w_{12} \tanh \left(2 x_{2}\right) \\
& +\tanh \left(x_{3}\right)-5.5 \tanh \left(x_{4}\right) \\
\dot{x}_{2}= & -x_{2}-0.5 \tanh \left(2 x_{1}\right)+0.75 \tanh \left(2 x_{2}\right) \\
& +3.5 \tanh \left(x_{3}\right)-0.25 \tanh \left(x_{4}\right) \\
\dot{x}_{3}= & -x_{3}+w_{31} \tanh \left(2 x_{1}\right)-4 \tanh \left(2 x_{2}\right) \\
& +1.8 \tanh \left(x_{3}\right)+4 \tanh \left(x_{4}\right) \\
\dot{x}_{4}= & -x_{4}+0.6 \tanh \left(2 x_{1}\right)+w_{43} \tanh \left(x_{3}\right) \\
& +2 \tanh \left(x_{4}\right)
\end{aligned}
$$

According to Equation (21), the small neural network circuit structure is designed in Fig.15(b). Four membrane potentials $x_{1}, x_{2}, x_{3}, x_{4}$ are emulated by four output voltages $v_{1}, v_{2}, v_{3}$, $v_{4}$, respectively. All synaptic weight coefficients are simulated by the resistors $R_{1}-R_{15}$. Based on the circuit in Fig.15(b), the circuit state equations can be described by

$$
\left\{\begin{aligned}
\frac{R C d v_{1}}{d t}=- & v_{1}+\frac{R}{R_{1}} \tanh \left(v_{1}\right)+\frac{R}{R_{2}} \tanh \left(v_{2}\right) \\
& +\frac{R}{R_{3}} \tanh \left(v_{3}\right)-\frac{R}{R_{4}} \tanh \left(v_{4}\right) \\
\frac{R C d v_{2}}{d t}= & -v_{2}-\frac{R}{R_{5}} \tanh \left(v_{1}\right)+\frac{R}{R_{6}} \tanh \left(v_{2}\right) \\
& +\frac{R}{R_{7}} \tanh \left(v_{3}\right)-\frac{R}{R_{8}} \tanh \left(v_{4}\right) \\
\frac{R C d v_{3}}{d t}= & -v_{3}+\frac{R}{R_{9}} \tanh \left(v_{1}\right)-\frac{R}{R_{10}} \tanh \left(v_{2}\right) \\
& +\frac{R}{R_{11}} \tanh \left(v_{3}\right)+\frac{R}{R_{12}} \tanh \left(v_{4}\right) \\
\frac{R C d v_{4}}{d t}= & -v_{4}+\frac{R}{R_{13}} \tanh \left(v_{1}\right)+\frac{R}{R_{14}} \tanh \left(v_{3}\right) \\
& +\frac{R}{R_{15}} \tanh \left(v_{4}\right)
\end{aligned}\right.
$$

Assume that $C_{1}=C_{2}=C_{3}=C_{4}=C, R C=10$ us, the resistance $R=10 \mathrm{k} \Omega$, then the $C$ can be chosen as $1 \mathrm{nF}$. Considering the fixed synaptic weight coefficients, relevant resistances can be calculated as $R_{1}=40 \mathrm{k} \Omega, R_{3}=10 \mathrm{k} \Omega, R_{4}=1.818 \mathrm{k} \Omega, R_{5}=20$ $\mathrm{k} \Omega, R_{6}=13.333 \mathrm{k} \Omega, R_{7}=2.857 \mathrm{k} \Omega, R_{8}=40 \mathrm{k} \Omega, R_{10}=2.5 \mathrm{k} \Omega$, $R_{11}=5.556 \mathrm{k} \Omega, R_{12}=2.5 \mathrm{k} \Omega, R_{13}=16.667 \mathrm{k} \Omega, R_{15}=5 \mathrm{k} \Omega$. Besides, $R_{2}=R / 0.5 w_{12}, R_{9}=R / w_{31}$, and $R_{14}=R / w_{43}$ are used to replace adjustable synaptic weight coefficients $w_{12}, w_{31}$, and $w_{43}$, respectively.

The designed small neural network circuit in Fig.15(b) is physically implemented on the experimental breadboard by using commercially available electronic elements including $\mathrm{R} /$ metal resistors and precision potentiometers, $C /$ ceramic capacitors, D/1N4007, U/TL082CP, and $\pm 15 \mathrm{~V}$ DC voltage supplies, as shown in Fig.16(f). Experimental results show that the designed neural network circuit can generate the results consistent with the numerical simulation results in Section III. For example, period-4 bursting in Fig.3(a), period-5 bursting in Fig.3(b), period-7 bursting in Fig.3(d), stochastic bursting in Fig.6(d), and chaotic bursting in Fig.6(e) are given in Fig.16(a)-(e), respectively. It is remarked that since there are 
parasitic parameters in the practical circuit, the resistances of resistors have some difference, which can be solved by finetuning the adjustable resistors.

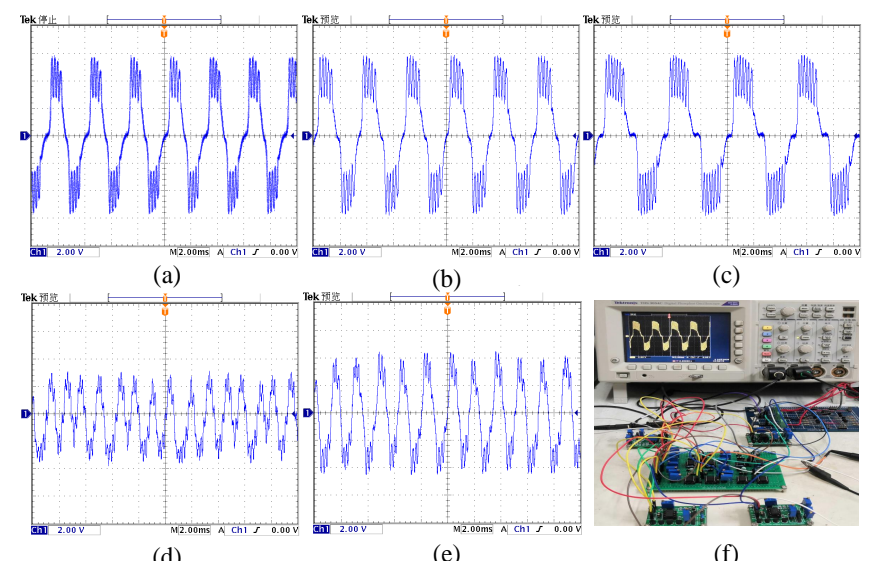

Fig. 16: Experimentally captured bursting firings from the neural network circuit. (a) period-4 bursting with $R_{14}=54.825$ $\mathrm{k} \Omega$. (b) period-5 bursting with $R_{14}=1 \mathrm{M} \Omega$. (c) period-7 bursting with $R_{14}=39.274 \mathrm{k} \Omega$. (d) stochastic bursting with $R_{9}=24.342 \mathrm{k} \Omega$. (e) chaotic bursting with $R_{9}=6.665 \mathrm{k} \Omega$. (f) hardware breadboard prototype for the small neural network.

\section{B. Realization and simulation of the coupling neural network circuit}

Based on the small neural network circuit in Fig.15(b), the circuit of the coupling neural network is designed as shown in Fig.17. It is noted that the coupling neural network circuit is composed of four parts: the first sub-network circuit in the yellow area, the second sub-network circuit in the blue area, the coupling circuit in the dark red area, and a controllable switch $S$. According to Equation (6), the circuit state equations of the coupling neural network circuit are given by

$$
\left\{\begin{array}{l}
\frac{R C d v_{x 1}}{d t}=-v_{x 1}+\frac{R}{R_{1}} \tanh \left(v_{x 1}\right)+\frac{R}{R_{2}} \tanh \left(v_{x 2}\right) \\
+\frac{R}{R_{3}} \tanh \left(v_{x 3}\right)-\frac{R}{R_{4}} \tanh \left(v_{x 4}\right)+\frac{R}{R_{L}}\left(v_{x 1}-v_{y 1}\right) \\
\frac{R C d v_{x 2}}{d t}=-v_{x 2}-\frac{R}{R_{5}} \tanh \left(v_{x 1}\right)+\frac{R}{R_{6}} \tanh \left(v_{x 2}\right) \\
\quad+\frac{R}{R_{7}} \tanh \left(v_{x 3}\right)-\frac{R}{R_{8}} \tanh \left(v_{x 4}\right) \\
\frac{R C d v_{x 3}}{d t}=-v_{x 3}+\frac{R}{R_{9}} \tanh \left(v_{x 1}\right)-\frac{R}{R_{10}} \tanh \left(v_{x 2}\right) \\
\quad+\frac{R}{R} \tanh \left(v_{x 3}\right)+\frac{R}{R_{12}} \tanh \left(v_{x 4}\right) \\
\frac{R C d v_{x 4}}{d t}=-v_{x 4}+\frac{R}{R_{13}} \tanh \left(v_{x 1}\right)-\frac{R}{R_{14}} \tanh \left(v_{x 3}\right) \\
+\frac{R}{R_{15}} \tanh \left(v_{x 4}\right) \\
\frac{R C d v_{y 1}}{d t}=-v_{y 1}+\frac{R}{R_{1}} \tanh \left(v_{y 1}\right)+\frac{R}{R_{2}} \tanh \left(v_{y 2}\right) \\
+\frac{R}{R_{3}} \tanh \left(v_{y 3}\right)-\frac{R}{R_{4}} \tanh \left(v_{y 4}\right)-\frac{R}{R_{L}}\left(v_{x 1}-v_{y 1}\right) \\
\frac{R C d v_{y 2}}{d t}=-v_{y 2}-\frac{R}{R_{5}} \tanh \left(v_{y 1}\right)+\frac{R}{R_{6}} \tanh \left(v_{y 2}\right) \\
+\frac{R}{R_{7}} \tanh \left(v_{y 3}\right)-\frac{R}{R_{8}} \tanh \left(v_{y 4}\right) \\
\frac{R C d v_{y 3}}{d t}=-v_{y 3}+\frac{R}{R_{9}} \tanh \left(v_{y 1}\right)-\frac{R}{R_{10}} \tanh \left(v_{y 2}\right) \\
+\frac{R}{R} \tanh \left(v_{y 3}\right)+\frac{R}{R_{12}} \tanh \left(v_{y 4}\right) \\
\frac{R C d v_{y 4}}{d t}=-v_{y 4}+\frac{R}{R_{13}} \tanh \left(v_{y 1}\right)-\frac{R}{R_{14}} \tanh \left(v_{y 3}\right) \\
\quad+\frac{R}{R R_{15}} \tanh \left(v_{y 4}\right)
\end{array}\right.
$$

where capacitor voltages $v_{x 1}, v_{x 2}, v_{x 3}, v_{x 4}, v_{y 1}, v_{y 2}, v_{y 3}$, and $v_{y 4}$ simulate membrane potentials $x_{1}, x_{2}, x_{3}, x_{4}, y_{1}, y_{2}, y_{3}$, and $y_{4}$, respectively. $R_{L}$ denotes the coupling strength, and it can be calculated as $R_{L}=R / \rho$.

Due to complex circuit structure, strong temperature drifts effect of circuit components, and large parameter difference between the same diode, it is difficult to physically implement the synchronization circuit. Therefore, to verify the availability of the coupling neural network circuit, the circuit in Fig.17 is realized and simulated in PSPICE. In the simulation process, the DC voltages of the operational amplifiers are set as $\pm 200 \mathrm{~V}$, and the controllable switch has two states: when $\rho<0,1$ and 2 connect to 4 and 3 , respectively, and when $\rho>0,1$ and 2 connect to 3 and 4, respectively. Extensive circuit simulation results show that the realized coupling neural network circuit can obtain the results consistent with the numerical simulation results in Section IV. For instance, complete bursting synchronization in Fig.13(a1), asynchronous bursting in Fig.13(a2), anti-phase bursting synchronization in Fig.13(a3), anti-phase bursting synchronization in Fig.13(a4), anti-phase spiking synchronization in Fig.13(a5), and unbounded state in Fig.13(a6) are given in Fig.18(a)-(f), respectively. It should be noted that the circuit simulation results are slightly different from the numerical results because of the computing errors between two different tools.

\section{CONCLUSION}

In this paper, we presented two neural network models that are capable of reproducing biological neural behaviors including bursting and synchronization. First, based on the original Hopfield neural network, a small neural network model with four neurons is established to generate various types of bursting firings such as periodic bursting with different spikes per burst, stochastic bursting, chaotic bursting, coexisting stochastic and chaotic burstings, as well as periodic and chaotic burstings with different amplitudes. Next, by emulating the synaptic coupled mechanism between two biological neural systems, we constructed a synaptic coupling neural network model using two coupled samll neural networks. By using Lyapunov stability theory and constructing a proper Lyapunov function, a set of sufficient conditions are derived to prove the synchronization ability of the two coupled neural networks. In particular, the numerical simulation results show that this coupling neural network model can exhibit abundant synchronization phenomena including complete bursting synchronization, anti-phase bursting synchronization, and antiphase spiking synchronization. Finally, we designed and implemented two neural network circuits by using commercially available electric elements. And some experimental results have been given to verify the effectiveness of the theoretical analyses and numerical results.

Although there are conflicting views about the role of dynamical behaviors in the functioning of the brain, modeling, analysis, simulation, and implementation of the brain neural system as attempted in this work will be helpful in clinical aspects and artificial intelligence applications. In future work, we will devote to study the brain-like dynamics of the largescale neural networks which are more similar to the real biological neural systems. We will also explore the practical applications of the brain-like neural network developed here. 


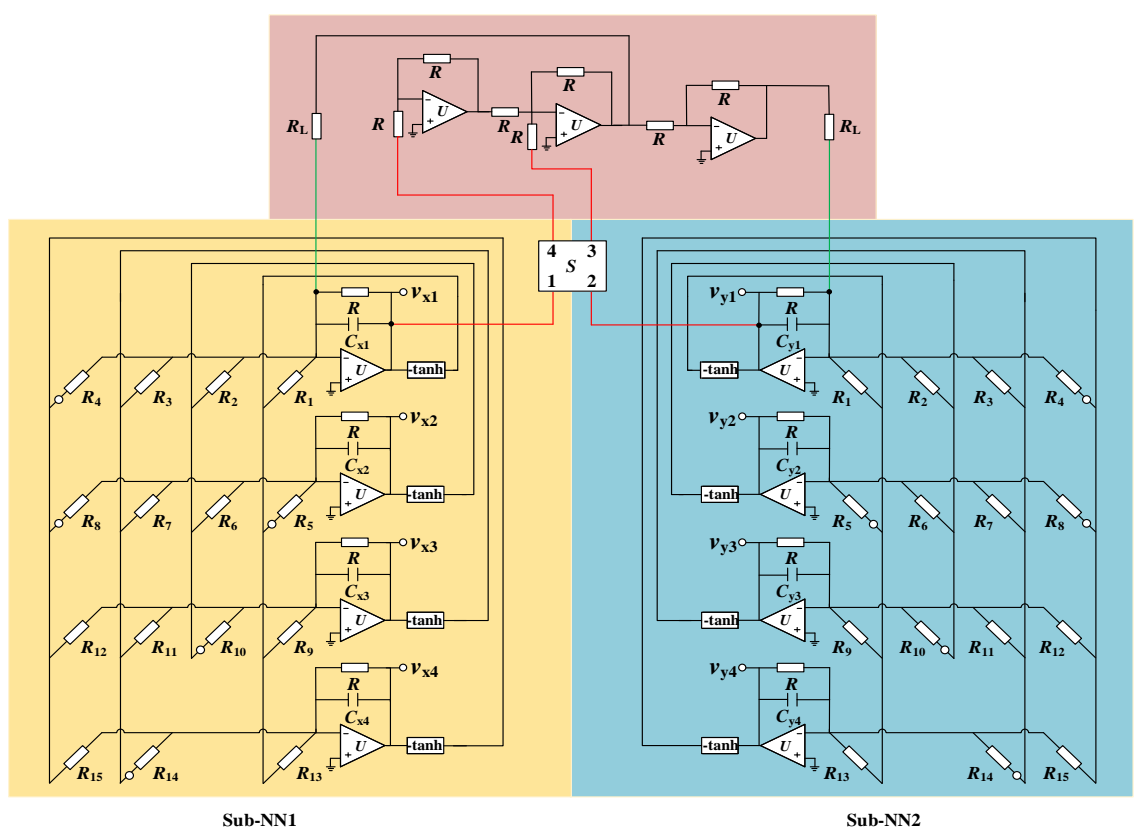

Fig. 17: The coupling neural network circuit.

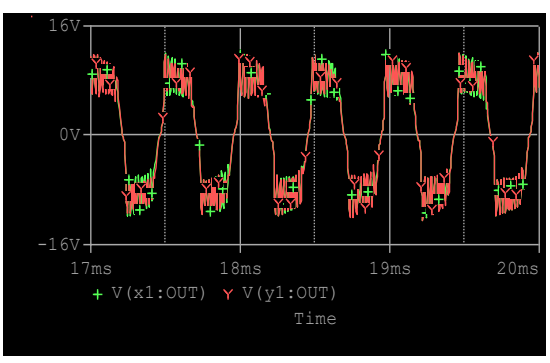

(a)

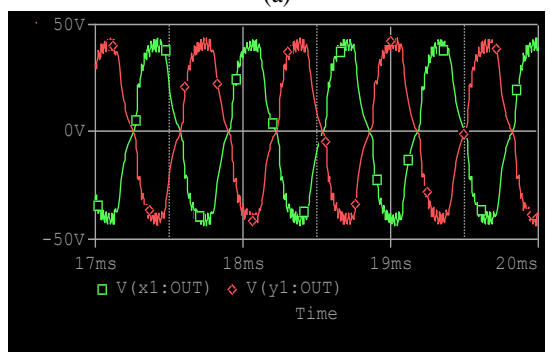

(d)

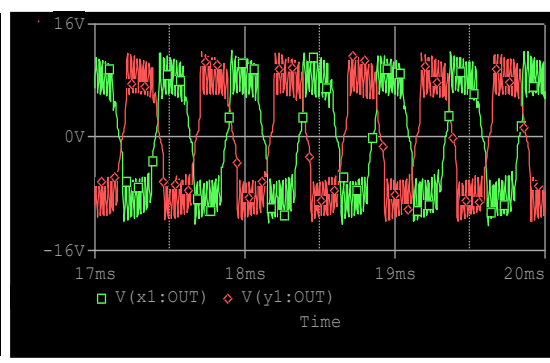

(b)

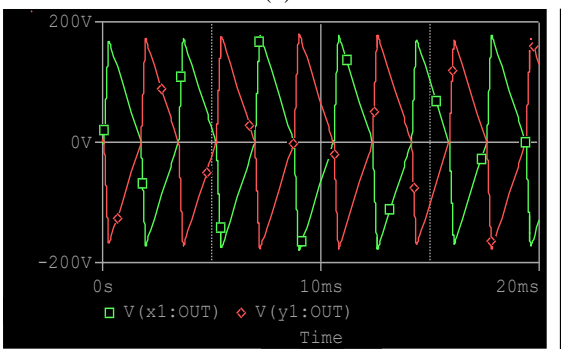

(e)

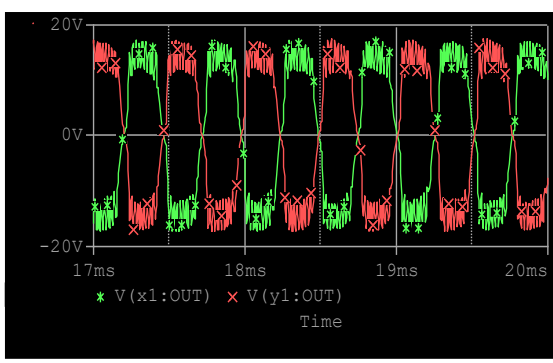

(c)

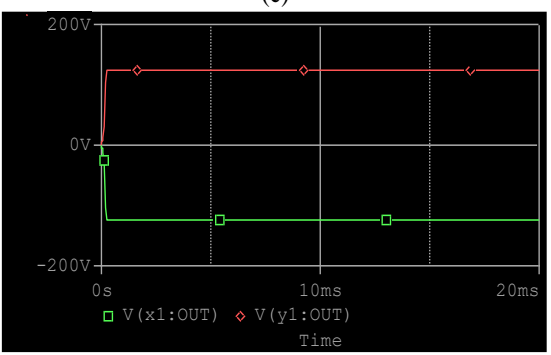

(f)

Fig. 18: PSPICE simulation results of the coupling neural network circuit. (a) complete bursting synchronization with $R_{L}=5$ $\mathrm{k} \Omega$. (b) asynchronous bursting with $R_{L}=1000 \mathrm{k} \Omega$. (c) anti-phase bursting synchronization with $R_{L}=50 \mathrm{k} \Omega$. (d) anti-phase bursting synchronization with $R_{L}=25 \mathrm{k} \Omega$ (e) anti-phase spiking synchronization with $R_{L}=20 \mathrm{k} \Omega$. (f) unbounded state with $R_{L}=19.5 \mathrm{k} \Omega$.

\section{REFERENCES}

[1] J. J. Hopfield, "Neural network and physical system with emergent collective computational abilities," Proc. Nat. Acad. Sc., vol. 79, pp. 25542558, 1982.

[2] E. Bullmore and O. Sporns "Complex brain networks: graph theoretical analysis of structural and functional systems," Nat. Rev. Neurosci., vol. 10, no. 3, pp. 186-198, 2009.

[3] W. Y. Hsu, "Application of competitive Hopfield neural network to braincomputer interface systems," Int. J. Neural Syst., vol. 22, no. 1, pp. 51-62, 2012.

[4] J. Yang, L. Wang, Y. Wang, and T. Guo, "A novel memristive Hopfield neural network with application in associative memory,"Neurocomputing., vol. 227, pp. 142-148, 2017.
[5] O. Krestinskaya, et al., "Learning in memristive neural network architecures using analog backpropagation circuits," IEEE Trans. Circuits Syst. I-Regul. Pap., vol. 66, no. 2, pp. 719-732, 2019.

[6] Q. Hong, Y. Li, and X. Wang, "Memristive continuous Hopfield neural network circuit for image restoration," Neural Comput. Appl., vol. 32, no. 12, pp. 8175-8185, 2020.

[7] H. Bersini, "The frustrated and compositional nature of chaos in small Hopfield networks," Neural Netw., vol. 11, no. 6, pp. 1017-1025, 1998.

[8] P. Zheng, W. Tang, and J. Zhang, "Letters: some novel double-scroll chaotic attractors in Hopfield networks," Neurocomputing., vol. 73, no. 10, pp. 2280-2285, 2010.

[9] B. Bao, et al., "Numerical analyses and experimental validations of coexisting multiple attractors in Hopfield neural network," Nonlinear Dyn., vol. 90, no. 4, pp. 2359-2369, 2017. 
[10] B. Bao, et al., "Dynamical effects of neuron activation gradient on Hopfield neural network: numerical analyses and hardware experiments," Int. J. Bifurcation Chaos., vol. 29, 2019, Art. no. 1930010.

[11] Y. Leng, et al., "Dynamic behaviors of hyperbolic-type memristor-based Hopfield neural network considering synaptic crosstalk," Chaos., vol. 30, 2020, Art. no. 33108.

[12] P. C. Rech, "Chaos and hyperchaos in a Hopfield neural network," Neurocomputing., vol. 74, no. 17, pp. 3361-3364, 2011.

[13] Q. Li, S. Tang, H. Zeng, and T. Zhou, "On hyperchaos in a small memristive neural network," Nonlinear Dyn., vol. 78, no. 2, pp. 1087-1099, 2014.

[14] Z. T. Njitacke, et al., "Extremely rich dynamics from hyperchaotic Hopfield neural network: Hysteretic dynamics, parallel bifurcation branches, coexistence of multiple stable states and its analog circuit implementation,” Eur. Phys. J.-Spec. Top., vol. 229, no. 6, pp. 1133-1154, 2020.

[15] V. T. Pham, et al., "A novel memristive neural network with hidden attractors and its circuitry implementation," Sci. China-Technol. Sci., vol. 59, no. 3, pp. 358-363, 2016.

[16] B. Bao, et al., "Coexisting behaviors of asymmetric attractors in hyperbolic-type memristor based Hopfield neural network," Front. Comput. Neurosci., vol. 11, 2017, Art. no. 81.

[17] C. Chen, "Coexisting multi-stable patterns in memristor synapse-coupled Hopfield neural network with two neurons," Nonlinear Dyn., vol. 95, no. 4, pp. 3385-3399, 2019.

[18] H. Lin, C. Wang, Q. Hong, Y. Sun, "A multi-stable memristor and its application in a neural network," IEEE Trans. Cir. Sys.-II: Brief Papers., vol. 67 , no. 12, pp. 3472-3476, 2020.

[19] X. Hu, et al., "Chaotic dynamics in a neural network under electromagnetic radiation," Nonlinear Dyn., vol. 91, no. 3, pp. 1541-1554, 2018.

[20] H. Lin and C. Wang, "Influences of electromagnetic radiation distribution on chaotic dynamics of a neural network," Appl. Math. Comput., vol. 369, 2020, Art. no. 124840.

[21] H. Lin, C. Wang, and Y. Tan, "Hidden extreme multistability with hyperchaos and transient chaos in a Hopfield neural network affected by electromagnetic radiation," Nonlinear Dyn., vol. 99, no. 3, pp. 2369-2386, 2020.

[22] H. Lin, C. Wang, W. Yao, and Y. Tan, "Chaotic dynamics in a neural network with different types of external stimuli," Commun. Nonlinear Sci. Numer. Simul., vol. 90, 2020, Art. no. 105390.

[23] D. M. Suter, et al., "Mammalian genes are transcribed with widely different bursting kinetics," Science., vol. 332, no. 6028, pp. 472-474, 2011.

[24] J. Wang, B. Lu, S. Liu, and X. Jiang, "Bursting types and bifurcation analysis in the pre-bötzinger complex respiratory rhythm neuron," Int. J. Bifurcation Chaos., vol. 27, 2017, Art. no. 1750010.

[25] H. Lin, C. Wang, Y. Sun, and W. Yao, "Firing multistability in a locally active memristive neuron model," Nonlinear Dyn., vol. 100, no. 4, pp. 3667-3683, 2020.

[26] H. Bao, A. Hu, W. Liu, and B. Bao, "Hidden bursting firings and bifurcation mechanisms in memristive neuron model with threshold electromagnetic induction," IEEE Trans. Neural Netw. Learn. Syst., vol. 31 , no. 2 , pp. 502-511, 2020.

[27] M. Hayati, M. Nouri, S. Haghiri, and D. Abbott, "Digital multiplierless realization of two coupled biological Morris-Lecar neuron model," IEEE Trans. Circuits Syst. I-Regul. Pap., vol. 62, no. 7, pp. 1805-1814, 2015.

[28] B. Bao, et al., "Chaotic bursting dynamics and coexisting multistable firing patterns in 3D autonomous Morris-Lecar model and microcontrollerbased validations," Int. J. Bifurcation Chaos., vol. 29, 2019, Art. no. 1950134.

[29] H. Soleimani and E. M. Drakakise, "An efficient and reconfigurable synchronous neuron model," IEEE Trans. Circuits Syst. II-Express Briefs., vol. 65 , no. 1, pp. 91-95, 2018.

[30] F. Zhu, R. Wang, K. Aihara, and X. Pan, "Energy-efficient firing patterns with sparse bursts in the Chay neuron model," Nonlinear Dyn., vol. 100, no. 3, pp. 2657-2672, 2020.

[31] Q. Xu, et al., "Two-neuron-based non-autonomous memristive Hopfield neural network: Numerical analyses and hardware experiments," AEU-Int. J. Electron. Commun., vol. 96, pp. 66-74, 2018.

[32] Z. T. Njitacke, C. L. Matze, M. F. Tsotsop, and J. Kengne, "Remerging feigenbaum trees, coexisting behaviors and bursting oscillations in a novel 3D generalized Hopfield neural network," Neural Process. Lett., vol. 52, no. 1, pp. 267-289, 2020.

[33] Z. T. Njitacke, J. Kengne, and H. B. Fotsin, "Coexistence of multiple stable states and bursting oscillations in a 4D Hopfield neural network," Circuits Syst. Signal Process., vol. 39, no. 7, pp. 3424-3444. 2020.
[34] C. M. Gray, "Synchronization oscillations in neuronal systems: mechanisms and functions," J. Comput. Neurosci., vol. 1, nos. 1-2, pp. 11-38, 1994.

[35] E. Rodriguez, et al., "Perception's shadow: long-distance synchronization of human brain activity," Nature., vol. 397, no. 6718, pp. 430-433, 1999.

[36] P. J. Uhlhaas and W. Singer, "Neural synchrony in brain disorders: relevance for cognitive dysfunctions and pathophysiology," Neuron., vol. 52, no. 1, pp. 155-168, 2006.

[37] L. Zhou, C. Wang, and L. Zhou, "Cluster synchronization on multiple sub-networks of complex networks with nonidentical nodes via pinning control," Nonlinear Dyn., vol. 83, no. 1, pp. 1079-1100, 2016.

[38] P. Liu, Z. Zeng, and J. Wang, "Global synchronization of coupled fractional-order recurrent neural networks," IEEE Trans. Neural Netw. Learn. Syst., vol. 30, no. 8, pp. 2358-2368, 2019.

[39] W. Yao, et al., "Exponential multistability of memristive CohenGrossberg neural networks with stochastic parameter perturbations," Appl. Math. Comput., vol. 386, 2020, Art. no. 125483.

[40] Z. Zhang and J. Cao, "Novel finite-time synchronization criteria for inertial neural networks with time delays via integral inequality method," IEEE Trans. Neural Netw. Learn. Syst., vol. 30, no. 5, pp. 1476-1485, 2019.

[41] W. Yao, C. Wang, Y. Sun, and C. Zhou, "Robust multimode function synchronization of memristive neural networks with parameter perturbations and time-varying delays," IEEE Trans. Syst. Man Cybern. -Syst., 2020, doi: 10.1109/TSMC.2020.2997930.

[42] F. Xu, et al., "Synchronous dynamics in neural system coupled with memristive synapse," Nonlinear Dyn., vol. 92, no. 3, pp. 1395-1402, 2018.

[43] A. S. Etémé, C. B. Tabi, and A. Mohamadou, "Firing and synchronization modes in neural network under magnetic stimulation," Commun. Nonlinear Sci. Numer. Simul., vol. 72, pp. 432-440, 2019.

[44] B. Bao, et al., "Initial-induced coexisting and synchronous firing activities in memristor synapse-coupled Morris-Lecar bi-neuron network," Nonlinear Dyn., vol. 99, no. 3, pp. 2339-2354, 2020.

[45] F. Parastesh, et al., "Synchronizability of two neurons with switching in the coupling," Appl. Math. Comput., vol. 350, pp. 217-223, 2019.

[46] X. Hu and C. Liu, 'Bursting and synchronization of coupled neurons under electromagnetic radiation," Complexity., vol. 2019, 2019, Art. no. 4835379.

[47] H. Bao, Y. Zhang, W. Liu, and B. Bao, "Memristor synapse-coupled memristive neuron network: synchronization transition and occurrence of chimera," Nonlinear Dyn., vol. 100, no. 1, pp. 937-950, 2020.

[48] N. Wang, et al., "Generating multi-scroll Chua's attractors via simplified piecewise-linear Chua's diode," IEEE Trans. Circuits Syst. I-Regul. Pap., vol. 66, no. 12, pp. 4767-4779, 2019.

[49] H. R. Lin, et al., "An extremely simple multi-wing chaotic system: dynamics analysis, encryption application and hardware implementation," IEEE Trans. Ind. Electron.,2020, doi:10.1109/TIE.2020.3047012.

[50] S. Lakshmanan, et al., "Dynamical analysis of the Hindmarsh-Rose neuron with time delays," IEEE Trans. Neural Netw. Learn. Syst., vol. 28, no. 8, pp. 1953-1958, 2017.

[51] Z. Galias, "Study of amplitude control and dynamical behaviors of a memristive band pass filter circuit," IEEE Trans.Cir. Sys.-II: Brief Papers., vol. 65 , no. 6 , pp. 637-641, 2018.

[52] X. Zhang, et al., "A memristive chaotic oscillator with controllable amplitude and frequency," Chaos Solitons Fractals., vol. 139, 2020, Art. no. 110000

[53] W. Liu, J. Xiao, X. Qian, and J. Yang, "Antiphase synchronization in coupled chaotic oscillators,"Physical Review E., vol. 73, no. 5, 2006, Art. no. 57203.

[54] Y. Jiang, et al.,, "Design and hardware implementation of neuromorphic systems with RRAM synapses and threshold-controlled neurons for pattern recognition," IEEE Trans. Circuits Syst. I-Regul. Pap., vol. 65, no. 9, pp. 2726-2738, 2018.

[55] J. Wang, J. Lin, and Z. Wang, "Efficient hardware architectures for deep convolutional neural network," IEEE Trans. Circuits Syst. I-Regul. Pap., vol. 65, no. 6, pp. 1941-1953, 2018.

[56] A. Zahedi, S. Haghiri, and M. Hayati, "Multiplierless digital implementation of time-varying FitzHugh-Nagumo model," IEEE Trans. Circuits Syst. I-Regul. Pap., vol. 66, no. 7, pp. 2662-2670, 2019.

[57] A. A. Gilan, M. Emad, and B. Aizadeh, "FPGA-based implementation of a real-time object recognition system using convolutional neural network," IEEE Trans.Cir. Sys.-II: Brief Papers., vol. 67, no. 4, pp. 755-759, 2020.

[58] Y. Yamashita, Y. Nakamura, "A neuron circuit model with smooth nonlinear output function,"IEICE Proceedings Series., vol. 41, no. 17PM1, pp. 1-3, 2007. 


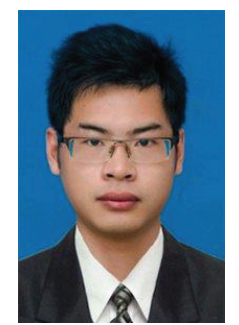

Hairong Lin received the B.S. degree in communication engineering from Huaihua University, Huaihua, China, in 2011, and the M.S. degree in information and communication engineering from Hunan University, Changsha, China, in 2015. He is currently working toward the Ph.D degree at the College of Computer Science and Electronic Engineering, Hunan University, Changsha, China.

$\mathrm{He}$ recently focuses on the modeling and analysis of neural systems, fundamental theory of nonlinear systems and circuits, and analog implementation of

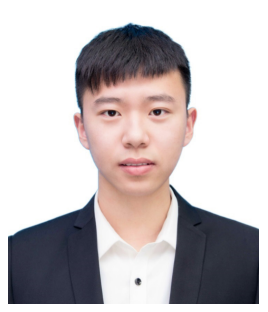

Chengjie Chen received the B.S. degree in Electrical engineering and its automation from the Huaiyin Normal University, Huaian, China, in 2018. He is currently pursuing the M.S. degree in electronics science and technology with the School of Microelectronics and Control Engineering, Changzhou University, Changzhou, China. His research interests include memristive neuromorphic circuit, and nonlinear circuits and systems.

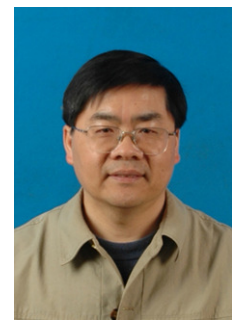

Chunhua Wang received the M.S. degree from Zhengzhou University, Zhengzhou, China, in 1994, and the Ph.D. degree from Beijing University of Technology, Beijing, China, in 2003.

$\mathrm{He}$ is currently the Professor of College of Information Science and Engineering, Hunan University, Changsha, China. He is the Doctor tutor, director of advanced communication technology key laboratory of hunan universities, the member of academic committee of hunan university, the director of chaos and nonlinear circuit professional committee of circuit and system branch of China electronic society. Now, his research interests include memristor circuit, complex networks, chaotic circuit, chaos secure communication, current-mode circuit and neural networks based on memristor. He has presided over 8 national and provincial projects, and published more than 120 papers, among which more than 100 were retrieved by SCI.

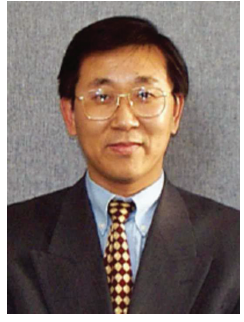

Yichuang Sun (M'90-SM'99) received the B.Sc. and M.Sc. degrees from Dalian Maritime University, Dalian, China, in 1982 and 1985, respectively, and the Ph.D. degree from the University of York, York, U.K., in 1996, all in communications and electronics engineering.

Dr. Sun is currently Professor of Communications and Electronics, Head of Communications and Intelligent Systems Research Group, and Head of Electronic, Communication and Electrical Engineering Division in the School of Engineering and Computer Science of the University of Hertfordshire, UK. He has published over 330 papers and contributed 10 chapters in edited books. He has also published four text and research books: Continuous-Time Active Filter Design (CRC Press, USA, 1999), Design of High Frequency Integrated Analogue Filters (IEE Press, UK, 2002), Wireless Communication Circuits and Systems (IET Press, 2004), and Test and Diagnosis of Analogue, Mixed-signal and RF Integrated Circuits - the Systems on Chip Approach (IET Press, 2008). His research interests are in the areas of wireless and mobile communications, RF and analogue circuits, microelectronic devices and systems, and machine learning and deep learning.

Professor Sun was a Series Editor of IEE Circuits, Devices and Systems Book Series (2003-2008). He has been Associate Editor of IEEE Transactions on Circuits and Systems I: Regular Papers (2010-2011, 2016-2017, 20182019). He is also Editor of ETRI Journal, Journal of Semiconductors, and Journal of Sensor and Actuator Networks. He was Guest Editor of eight IEEE and IEE/IET journal special issues: High-frequency Integrated Analogue Filters in IEE Proc. Circuits, Devices and Systems (2000), RF Circuits and Systems for Wireless Communications in IEE Proc. Circuits, Devices and Systems (2002), Analogue and Mixed-Signal Test for Systems on Chip in IEE Proc. Circuits, Devices and Systems (2004), MIMO Wireless and Mobile Communications in IEE Proc. Communications (2006), Advanced Signal Processing for Wireless and Mobile Communications in IET Signal Processing (2009), Cooperative Wireless and Mobile Communications in IET Communications (2013), Software-Defined Radio Transceivers and Circuits for 5G Wireless Communications in IEEE Transactions on Circuits and Systems-II (2016), and the 2016 IEEE International Symposium on Circuits and Systems in IEEE Transactions on Circuits and Systems-I (2016). He has also been widely involved in various IEEE technical committee and international conference activities.

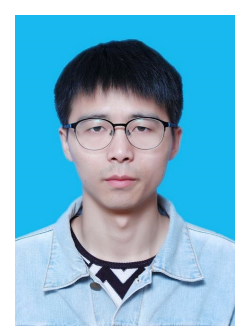

Chao Zhou received the B.S. degree in automation from the Xi'an University of Science and Technology, Xi'an, China, in 2014, and the M.S. degree in control science and engineering from Hunan University, Changsha, China, in 2017, where he is currently pursuing the Ph.D. degree in computer science and technology. His current research interests include the nonlinear dynamical systems, complex systems and memristive neural networks.

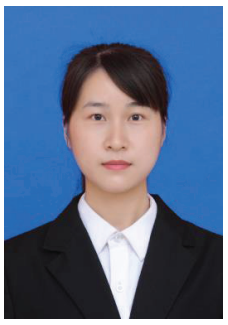

Cong Xu received the Bachelor's degree in communications engineering from Hunan City University, Yiyang, China. She is currently pursuing the $\mathrm{Ph}$. D degree in College of Computer Science and Electronic Engineering, Hunan University, China. Her research interests include memristor neural network and its applications.

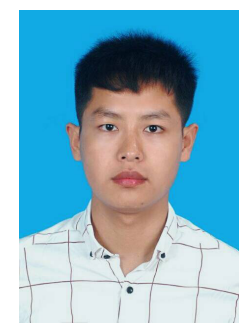

Intelligence.
Qinghui Hong received the B.S degree and M.S degree in electronic science and technology from Xiangtan University, Xiangtan, China, in 2012 and 2015 , respectively, and the Ph.D degree in computer system architecture from Huazhong University of Science and Technology, Wuhan, China, in 2019. He is currently an Assistant Professor with college of computer science and electronic engineering, Hunan University, Changsha 410082, China.

His current research interests include memristive neural network and its application to Artifificial 